\title{
Higher derivative supersymmetric nonlinear sigma models on Hermitian symmetric spaces and BPS states therein
}

\author{
Muneto $\mathrm{Nitta}^{1, *}$ and Shin Sasaki ${ }^{2, \dagger}$ \\ ${ }^{1}$ Department of Physics, and Research and Education Center for Natural Sciences, Keio University, \\ Hiyoshi 4-1-1, Yokohama, Kanagawa 223-8521, Japan \\ ${ }^{2}$ Department of Physics, Kitasato University, Sagamihara 252-0373, Japan
}

(Received 19 November 2020; accepted 14 December 2020; published 4 January 2021)

\begin{abstract}
We formulate four-dimensional $\mathcal{N}=1$ supersymmetric nonlinear sigma models on Hermitian symmetric spaces with higher derivative terms, free from the auxiliary field problem and the Ostrogradski's ghosts, as gauged linear sigma models. We then study Bogomol'nyi-Prasad-Sommerfield equations preserving $1 / 2$ or $1 / 4$ supersymmetries. We find that there are distinct branches, that we call canonical $(F=0)$ and noncanonical $(F \neq 0)$ branches, associated with solutions to auxiliary fields $F$ in chiral multiplets. For the $\mathbb{C} P^{N}$ model, we obtain a supersymmetric $\mathbb{C} P^{N}$ Skyrme-Faddeev model in the canonical branch while in the noncanonical branch the Lagrangian consists of solely the $\mathbb{C} P^{N}$ Skyrme-Faddeev term without a canonical kinetic term. These structures can be extended to the Grassmann manifold $G_{M, N}=S U(M) /[S U(M-N) \times S U(N) \times U(1)]$. For other Hermitian symmetric spaces such as the quadric surface $\left.Q^{N-2}=S O(N) /[S O(N-2) \times U(1)]\right)$, we impose F-term (holomorphic) constraints for embedding them into $\mathbb{C} P^{N-1}$ or Grassmann manifold. We find that these constraints are consistent in the canonical branch but yield additional constraints on the dynamical fields, thus reducing the target spaces in the noncanonical branch.
\end{abstract}

DOI: 10.1103/PhysRevD.103.025001

\section{INTRODUCTION}

Nonlinear sigma models are typical examples of lowenergy effective theories. When a global symmetry $G$ is spontaneously broken down to its subgroup $H$, there appear massless Nambu-Goldstone bosons dominant at low energy, and their low-energy dynamics can be described by nonlinear sigma model whose target space is a coset space $G / H[1,2]$. If one wants to study physics at higher energy, one needs higher derivative correction terms, typically appearing in the chiral perturbation theory of QCD [3]. Other examples of higher derivative terms can be found in various contexts such as the Skyrme model, lowenergy effective theories of superstring theory, and world volume theories of topological solitons and branes such as Nambu-Goto and Dirac-Born-Infeld actions.

In supersymmetric theories, higher derivative terms often bring us troubles. It is known that constructing derivative terms in the form of $\partial_{m} \Phi$ suffers from a technical issue

\footnotetext{
*nitta@phys-h.keio.ac.jp

shin-s@kitasato-u.ac.jp
}

Published by the American Physical Society under the terms of the Creative Commons Attribution 4.0 International license. Further distribution of this work must maintain attribution to the author(s) and the published article's title, journal citation, and DOI. Funded by SCOAP ${ }^{3}$. called the auxiliary field problem $[4,5]$. This stems from the fact that the equation of motion for the auxiliary field $F$ in the chiral superfield $\Phi$ ceases to be an algebraic equation and becomes a kinematical one. As a consequence, it is hard to integrate out the auxiliary field and the interactions of physical fields are not apparent. Supersymmetric extensions of the Wess-Zumino-Witten term [6] and the SkyrmeFaddeev model $[7,8]$ are such examples with the auxiliary field problem, while low-energy Lagrangians for supersymmetric gauge theories in Refs. [9-11] (see also Refs. [12-14]), and supersymmetric extensions of DiracBorn-Infeld action $[15,16]$ and K-field theories $[17,18]$ are free from this problem. A broad class of supersymmetric derivative terms free from the auxiliary field problem and the Ostrogradski's ghost [19] was found as four-dimensional $\mathcal{N}=1$ supersymmetric higher derivative chiral models formulated in terms of superfields [20-32]. ${ }^{1}$ The model consists of a Kähler potential $K$ and a superpotential $W$ together with a $(2,2)$ Kähler tensor $\Lambda$ that determines derivative corrections. It was applied to a new mechanism of supersymmetry breaking in modulated vacua [36] (see also Ref. [37]). The general formulation was extended for other superfields: the most general ghost-free

\footnotetext{
${ }^{1}$ Another possibility is to gauge them away [33] if there are Ostrogradski's higher derivative ghosts $[34,35]$.
} 
(and tachyon-free) higher derivative $\mathcal{N}=1$ supersymmetric Lagrangian for vector multiplets (1-form gauge fields) [38] and 3-form gauge field [39]. As for nonlinear sigma models of chiral superfields, only known examples are the supersymmetric $\mathbb{C} P^{1}$ model (or baby Skyrme model) $[24,25,27,40-42]$ and the supersymmetric Skyrme model [30]. When one solves the auxiliary field equations of motion for the auxiliary field $F$, it in general allows more than one solution $F=0$ and $F \neq 0$. The former is called the canonical branch and the latter the noncanonical branch. The bosonic part of the Lagrangian of the canonical branch consists of a canonical kinetic term and a fourderivative term for the $\mathbb{C} P^{1}$ model [43]. This admits lump (sigma model instanton) solutions identical to those in the $\mathbb{C} P^{1}$ model without a higher derivative term [44], as Bogomol'nyi-Prasad-Sommerfield (BPS) states preserving $1 / 2$ supersymmetric charges among the original supersymmetry [27]. On the other hand, in the noncanonical branch, the bosonic part of the Lagrangian consists of only the fourderivative term of the Skyrme-Faddeev type [43] (that is without fourth order time derivatives) without any canonical kinetic term. This admits a compact baby Skyrmion [45] which is a BPS state preserving 1/4 supersymmetry [27]. While the general Lagrangian in superspace [20] can be defined on any Kähler target spaces, solving auxiliary field equations consisting of more than one chiral multiplet is still an open problem, except for a single matrix chiral superfield [30].

The purpose of this paper is to present higher derivative supersymmetric nonlinear sigma models with a wider class of target spaces-Hermitian symmetric spaces,

$$
\begin{aligned}
& \mathbb{C} P^{N-1}=S U(N) /[S U(N-1) \times U(1)], \\
& G_{M, N}=U(M) /[U(M-N) \times U(N)], \\
& Q^{N-2}=S O(N) /[S O(N-2) \times U(1)], \\
& S O(2 N) / U(N), \quad S p(N) / U(N), \\
& E_{6} /[S O(10) \times U(1)], \quad E_{7} /\left[E_{6} \times U(1)\right],
\end{aligned}
$$

for which we solve auxiliary field equations. In the case without higher derivative terms, supersymmetric nonlinear sigma models on Hermitian symmetric spaces can be constructed by imposing supersymmetric constraints on gauged linear sigma models [27]. This formulation is found to help us to solve auxiliary field equations even with higher derivative terms. We employ the gauged linear sigma models with higher derivative terms for chiral multiplets [29] and then take the strong gauge coupling (nonlinear sigma model) limit. Solving auxiliary field equations yields the canonical and noncanonical branches for the on-shell actions. For the $\mathbb{C} P^{N}$ model, we obtain a supersymmetric extension of the $\mathbb{C} P^{N}$ Skyrme-Faddeev model [46-49] in the canonical branch, while the on-shell Lagrangian in the noncanonical branch consists solely of the $\mathbb{C} P^{N}$ Skyrme-Faddeev term (the term without fourth order time derivatives). We then study BPS states in these models and find that lumps (sigma model instantons) identical to those without higher derivative terms remain 1/2 BPS states in the canonical branch, while compact baby skyrmions are 1/4 BPS states in the noncanonical branch.

The organization of this paper is as follows. In the next section, we introduce the supersymmetric higher derivative term in the gauged chiral models. We show that a derivative term determined by a $(2,2)$ Kähler tensor provides supersymmetric higher derivative terms free from the auxiliary field problem. We will see that there are two on-shell branches associated with the solutions to the auxiliary field in the chiral multiplets. In Sec. III, we study the sigma model limit of the gauged chiral models. We first focus on the sigma models whose target spaces are the Hermitian symmetric spaces $\mathbb{C} P^{N-1}$ and $G_{M, N}$. They are defined only by the D-term constraints. We examine the limit in the two distinct branches and write down the higher derivative terms in the nonlinear sigma models. In Sec. IV, we discuss the nonlinear sigma models defined by the F-term constraints in addition to D-term constraints, which include the target spaces $Q^{N-2}, \quad S O(2 N) / U(N), \quad S p(N) / U(N)$, $E_{6} /[S O(10) \times U(1)], E_{7} /\left[E_{6} \times U(1)\right]$. We will show that the F-term constraints give additional conditions on the target spaces in the nocanonical branch thus reducing the target spaces, while they do not in the canonical branch. This distinguishes the situation from the $\mathbb{C} P^{N-1}$ and $G_{M, N}$ cases.

In Sec. V, we discuss the BPS states in the nonlinear sigma models, and in Sec. VI, we mention fermionic terms in the models. Section VII is devoted to conclusion and discussions. As for a superfield notation, we follow the Wess-Bagger convention [50].

\section{SUPERSYMMETRIC HIGHER DERIVATIVE TERMS IN THE CHIRAL MODEL}

In this section, we briefly introduce the supersymmetric higher derivative term to the chiral model that is free from the auxiliary field problem. The Lagrangian is given by [20,29]

$$
\begin{aligned}
\mathcal{L}= & \int d^{4} \theta K\left(\Phi, \Phi^{\dagger}\right) \\
& +\frac{1}{16} \int d^{4} \theta \Lambda_{i \bar{j} k \bar{l}, a b}^{c d}\left(\Phi, \Phi^{\dagger}\right) D^{\alpha} \Phi^{i a} D_{\alpha} \Phi^{k b} \bar{D}_{\dot{\alpha}} \Phi_{c}^{\dagger \bar{j}} \bar{D}^{\dot{\alpha}} \Phi_{d}^{\dagger \dagger} \\
& +\left(\int d^{2} \theta W(\Phi)+\text { H.c. }\right),
\end{aligned}
$$

where $K\left(\Phi, \Phi^{\dagger}\right)$ is a Kähler potential, $W(\Phi)$ is a superpotential, and $\Lambda_{i j k \bar{l}}\left(\Phi, \Phi^{\dagger}\right)$ is a $(2,2)$ Kähler tensor defined by the chiral superfields $\Phi, \Phi^{\dagger}$ and their derivatives $D_{\alpha} \Phi$, $\bar{D}_{\dot{\alpha}} \Phi^{\dagger}, \partial_{m} \Phi$, and $\partial_{m} \Phi^{\dagger}$. We assume that the chiral superfields $\Phi^{i a}\left(i=1, \ldots, \operatorname{dim} G, a=1, \ldots, \operatorname{dim} G^{\prime}\right)$ belong to the fundamental representations of the global and gauge 
symmetries $G$ and $G^{\prime}$, respectively. The four-dimensional $\mathcal{N}=1$ chiral superfield is expanded, in the chiral basis $y^{m}=x^{m}+i \theta \sigma^{m} \bar{\theta}$, as

$$
\Phi^{i a}=\varphi^{i a}(y)+\theta \psi^{i a}(y)+\theta^{2} F^{i a}(y) .
$$

We stress that the pure bosonic component in the fourth derivative part

$$
D^{\alpha} \Phi^{i a} D_{\alpha} \Phi^{k b} \bar{D}_{\dot{\alpha}} \Phi_{c}^{\dagger \bar{j}} \bar{D}^{\dot{\alpha}} \Phi_{d}^{\dagger \bar{l}}
$$

saturates the Grassmann coordinates, and only the bosonic fields in the Kähler tensor $\Lambda_{i \bar{j} k \bar{l}}$ contribute to this pure bosonic term [20]. Indeed, the component expansion of the term (2.3) can be evaluated as

$$
\begin{aligned}
D^{\alpha} \Phi^{i \alpha} D_{\alpha} \Phi^{k b} \bar{D}_{\dot{\alpha}} \Phi_{c}^{\dagger \bar{j}} \bar{D}^{\dot{\alpha}} \Phi_{d}^{\dagger \bar{\dagger}} \\
=16 \theta^{2} \bar{\theta}^{2}\left[\left(\partial_{m} \varphi^{i a} \partial^{m} \varphi^{k b}\right)\left(\partial_{n} \bar{\varphi}_{c}^{\bar{j}} \partial^{n} \bar{\varphi}_{d}^{\bar{l}}\right)\right. \\
\quad-\frac{1}{2}\left(\partial_{m} \varphi^{i a} F^{k b}+F^{i a} \partial_{m} \varphi^{k b}\right)\left(\partial^{m} \bar{\varphi}_{c}^{\bar{j}} \bar{F}_{d}^{\bar{l}}+\bar{F}_{c}^{\bar{j}} \partial^{m} \bar{\varphi}_{d}^{\bar{l}}\right) \\
\left.\quad+F^{i a} \bar{F}_{c}^{\bar{j}} F^{k b} \bar{F}_{d}^{\bar{l}}\right]+\cdots
\end{aligned}
$$

Here $\cdots$ are terms involving fermions. Due to this remarkable structure, a large class of supersymmetric models with derivative corrections can be realized by the Lagrangian (2.1). For example, the $\mathcal{N}=1$ supersymmetric Dirac-BornInfeld model is given by the Kähler tensor (scalar) $[15,16]$

$$
\begin{aligned}
\Lambda\left(\Phi, \Phi^{\dagger}\right) & =\frac{1}{1+A+\sqrt{(1+A)^{2}-B}}, \quad A=\partial_{m} \Phi \partial^{m} \Phi^{\dagger}, \\
B & =\partial_{m} \Phi \partial^{m} \Phi \partial_{n} \Phi^{\dagger} \partial^{n} \Phi^{\dagger} .
\end{aligned}
$$

A supersymmetric Skyrme-Faddeev model is given by the Kähler metric $K_{\varphi \bar{\varphi}}\left(1+|\varphi|^{2}\right)^{-2}$ and the Kähler scalar [27],

$$
\begin{aligned}
\Lambda\left(\Phi, \Phi^{\dagger}\right)= & \left(\partial_{m} \Phi \partial^{m} \Phi \partial_{n} \Phi^{\dagger} \partial^{n} \Phi^{\dagger}\right)^{-1} \frac{1}{\left(1+\Phi \Phi^{\dagger}\right)^{4}} \\
& \times\left[\left(\partial_{m} \Phi^{\dagger} \partial^{m} \Phi\right)^{2}-\partial_{m} \Phi \partial^{m} \Phi \partial_{n} \Phi^{\dagger} \partial^{n} \Phi^{\dagger}\right] .
\end{aligned}
$$

The other examples include the Galileon inflation and the ghost condensation models [21,22] and a superconformal higher derivative nonlinear sigma model [51].

Now we make the global symmetry $G^{\prime}$ be gauged by introducing the $\mathcal{N}=1$ vector multiplet $V=$ $V^{\hat{a}} T^{\hat{a}}\left(\hat{a}=0,1, \ldots, \operatorname{dim} \mathcal{G}^{\prime}\right)$,

$V=-\left(\theta \sigma^{m} \bar{\theta}\right) A_{m}(x)+i \theta^{2} \bar{\theta} \bar{\lambda}(x)-i \bar{\theta}^{2} \theta \lambda(x)+\frac{1}{2} \theta^{2} \bar{\theta}^{2} D(x)$.

Here we have employed the Wess-Zumino gauge and $T^{\hat{a}}$ are the generators of $\mathcal{G}^{\prime}$ satisfying the normalization $\operatorname{Tr}\left(T^{\hat{a}} T^{\hat{b}}\right)=k \delta^{\hat{a}} \hat{b}$. In the derivative corrections, the vector multiplet is introduced in the gauge covariantization of the supercovariant derivative:,

$$
D_{\alpha} \Phi^{i a} \rightarrow \mathcal{D}_{\alpha} \Phi^{i a}=D_{\alpha} \Phi^{i a}+\left(\Gamma_{\alpha}\right)_{b}^{a} \Phi^{i b}
$$

The gauge superconnection $\Gamma_{\alpha}$ is defined by

$$
\left(\Gamma_{\alpha}\right)^{a}{ }_{b}=e^{-2 g V} D_{\alpha} e^{2 g V},
$$

where $g$ is the gauge coupling constant. The gauge invariant derivative term is given by a $(2,2)$ Kähler tensor of the following structure:

$$
\Lambda_{i \bar{j} k \bar{l}, a b}{ }^{c d}=\Lambda_{i \bar{j} k \bar{l}}\left(\Phi, \Phi^{\dagger}\right)\left(e^{2 g V}\right)_{a}^{c}\left(e^{2 g V}\right)_{b}^{d},
$$

where $\Lambda_{i \bar{j} k \bar{l}}$ is composed of gauge invariant quantities made of $\Phi, \Phi^{\dagger}, V$. These include the gauge covariant derivatives of the chiral superfields $\mathcal{D}_{\alpha} \Phi, \overline{\mathcal{D}}_{\dot{\alpha}} \Phi^{\dagger}$. We note that terms made of $\partial_{m} \Phi, \partial_{m} \Phi^{\dagger}$, which are allowed in the ungauged case, are forbidden in the gauged models due to supersymmetry and the gauge covariance. Then, the gauged Lagrangian can be written as

$$
\begin{aligned}
\mathcal{L}= & \int d^{4} \theta K\left(\Phi, \Phi^{\dagger}, V\right)-\frac{1}{16} \int d^{4} \theta \Lambda_{i j \bar{j} \bar{l}}\left(\Phi, \Phi^{\dagger}\right)\left(\overline{\mathcal{D}}_{\dot{\alpha}} \Phi^{\dagger \bar{j}} e^{2 g V} \mathcal{D}^{\alpha} \Phi^{i}\right)\left(\overline{\mathcal{D}}^{\dot{\alpha}} \Phi^{\dagger \bar{\imath}} e^{2 g V} \mathcal{D}_{\alpha} \Phi^{k}\right) \\
& \left.+\left(\int d^{2} \theta W(\Phi)+\text { H.c. }\right)+\frac{1}{16 k g^{2}} \operatorname{Tr}\left[\int d^{2} \theta W^{\alpha} W_{\alpha}+\text { (H.c. }\right)\right]-2 \kappa g \int d^{4} \theta \operatorname{Tr} V,
\end{aligned}
$$

where we have introduced the gauge kinetic and Fayet-Iliopoulos (FI) terms with the FI parameter $\kappa$. The superpotential and the FI term are necessary to impose the supersymmetric constraints. The bosonic part of the Lagrangian is given by [29] 


$$
\begin{aligned}
\mathcal{L}_{\text {boson }}= & -\frac{\partial^{2} K}{\partial \bar{\varphi}_{a}^{\bar{j}} \partial \varphi^{i b}} D_{m} \bar{\varphi}_{a}^{\bar{j}} D^{m} \varphi^{i b}-\frac{\partial^{2} K}{\partial \bar{\varphi}_{a}^{\bar{j}} \partial \varphi^{i b}} \bar{F}_{a}^{\bar{j}} F^{i b}+\frac{g}{2} D^{\hat{a}}\left(\bar{\varphi}_{c}^{\bar{j}}\left(T^{\hat{a}}\right)^{c}{ }_{d} \frac{\partial K}{\partial \bar{\varphi}_{d}^{j}}+\frac{\partial K}{\partial \varphi^{i c}}\left(T^{\hat{a}}\right)^{c}{ }_{d} \varphi^{i d}-2 \kappa \delta^{\hat{a}}{ }_{0}\right) \\
& +\frac{1}{k} \operatorname{Tr}\left[-\frac{1}{4} F_{m n} F^{m n}+\frac{1}{2} D^{2}\right]+\frac{\partial W}{\partial \varphi^{i a}} F^{i a}+\frac{\partial \bar{W}}{\partial \bar{\varphi}_{a}^{i}} \bar{F}_{a}^{\bar{i}} \\
& +\Lambda_{i k \bar{j} \bar{l}}(\varphi, \bar{\varphi})\left[\left(D^{m} \bar{\varphi}_{a}^{\bar{j}} D^{n} \varphi^{i a}\right)\left(D_{m} \bar{\varphi}_{b}^{\bar{l}} D_{n} \varphi^{k b}\right)\right. \\
& \left.-\frac{1}{2}\left(D_{m} \varphi^{i a} F^{k b}+F^{i a} D_{m} \varphi^{k b}\right)\left(D^{m} \bar{\varphi}_{a}^{\bar{j}} \bar{F}_{b}^{\bar{l}}+\bar{F}_{a}^{\bar{j}} D^{m} \bar{\varphi}_{b}^{\bar{l}}\right)+F^{i a} \bar{F}_{a}^{\bar{j}} F^{k b} \bar{F}_{b}^{\bar{l}}\right],
\end{aligned}
$$

where $F_{m n}=\partial_{m} A_{n}-\partial_{m} A_{n}+i\left[A_{m}, A_{n}\right]$ and $T^{0}$ is the $U(1)$ generator in $G^{\prime}$. A notable fact about the Lagrangian (2.12) is that, due to the derivative corrections, the equation of motion for the auxiliary field $F$ is not linear,

$$
\begin{aligned}
& -\frac{\partial^{2} K}{\partial \bar{\varphi}_{a}^{\bar{j}^{\prime}} \partial \varphi^{i b}} F^{i b}+\frac{\partial \bar{W}}{\partial \bar{\varphi}_{a}^{\bar{j}^{\prime}}}+\Lambda_{i k \bar{j} \bar{l}}(\varphi, \bar{\varphi})\left[-\frac{1}{2}\left(\left(D_{m} \varphi^{i b} F^{k a}+F^{i b} D_{m} \varphi^{k a}\right)\right)\left(D^{m} \varphi_{b}^{\bar{j}} \delta^{\bar{j}^{\prime} \bar{l}}+\delta^{j^{\prime} \bar{j}} D^{m} \bar{\varphi}_{b}^{\bar{l}}\right)\right. \\
& \left.+F^{i a} \delta^{\bar{j}^{\prime} \bar{j}} F^{k b} \bar{F}_{b}^{\bar{l}}+F^{i b} \bar{F}_{b}^{\bar{j}} F^{k a} \delta^{j^{\prime} \bar{l}}\right]=0 .
\end{aligned}
$$

This apparently allows several solutions for the auxiliary field. The solutions provide several distinct on-shell Lagrangians. For example, when $W=0$, the inhomogeneous term in (2.13) vanishes and it is obvious that $F=0$ is a solution. We call this the canonical branch. In addition, we have another solution $F \neq 0$ and we call the corresponding theory the noncanonical branch. Both the branches exhibit remarkable features [16,23,26,27]. Although this shows an unusual situation in supersymmetric theories, we stress that Eq. (2.13) is the algebraic equation, not the kinematical one, which guarantees that $F$ still keeps the role of the auxiliary field.

In the following sections, we examine the nonlinear sigma model limit $g \rightarrow \infty$ in the gauged linear sigma models. In the limit, the vector multiplet carries nonpropagating degrees of freedom and it will be integrated out. We in particular focus on the Hermitian symmetric spaces of the type $G / H$ [52]. This procedure makes us to write down a variety of supersymmetric nonlinear sigma models with higher derivative terms.

\section{NONLINEAR SIGMA MODELS WITH D-TERM CONSTRAINTS}

In this section, we discuss supersymmetric nonlinear sigma models whose target spaces are the complex projective space $\mathbb{C} P^{N-1}$ and the Grassmann manifold $G_{M, N}$. It is known that they are obtained in the sigma model limit $g \rightarrow \infty$ of supersymmetric gauge theories with D-term constraints [52-54]. The former is obtained from an Abelian gauge theory, while the latter comes from a non-Abelian gauge theory. In the following, we explicitly integrate out the gauge field both in the canonical and the noncanonical branches in the sigma model limit $g \rightarrow \infty$.

\section{A. $\mathbb{C} P^{N-1}$ model}

We first consider the nonlinear sigma model whose target space is $\mathbb{C} P^{N-1}=S U(N) /[S U(N-1) \times U(1)]$. We formulate this model as a gauged linear sigma model with the global symmetry $G=S U(N)$ and gauge symmetry $G^{\prime}=U(1)$, where the latter is complexified $G_{\mathbb{C}}^{\prime}=U(1)_{\mathbb{C}}$. We consider the flat Kähler potential $K=\delta_{i \bar{j}} \Phi^{i} \Phi^{\dagger \bar{j}}=$ $\Phi^{\dagger i} \Phi^{i}$ and $W=0$. The chiral superfields $\Phi^{i}, \Phi^{\dagger i},(i=$ $1, \ldots, N)$ belong to the fundamental representation $\mathbf{N}$ of the global $S U(N)$ symmetry, and their $U(1)$ charges are assigned as $(+1,-1)$. We assume that the $(2,2)$ Kähler tensor is given by the flat metric as

$$
\Lambda_{i k \bar{j} \bar{l}}=\delta_{i \bar{j}} \delta_{k \bar{l}} \Lambda\left(\Phi, \Phi^{\dagger}\right),
$$

where $\Lambda(\Phi, \bar{\Phi})$ is a gauge invariant Kähler scalar composed of the chiral superfields and their gauge covariant derivatives $\mathcal{D}_{\alpha} \Phi, \overline{\mathcal{D}}_{\dot{\alpha}} \Phi^{\dagger}$. 2 Then, the Lagrangian becomes [53]

$$
\begin{aligned}
\mathcal{L}= & \int d^{4} \theta \Phi^{\dagger i} e^{2 V} \Phi^{i} \\
& +\frac{1}{16} \int d^{4} \theta \Lambda\left(\Phi, \Phi^{\dagger}\right) \delta_{i \bar{j}} \delta_{k \bar{l}} e^{4 V} \mathcal{D}^{\alpha} \Phi^{i} \mathcal{D}_{\alpha} \Phi^{k} \overline{\mathcal{D}}_{\dot{\alpha}} \Phi^{\dagger j} \overline{\mathcal{D}^{\alpha}} \Phi^{\dagger \bar{l}} \\
& \left.+\frac{1}{16 g^{2}}\left[\int d^{2} \theta W^{\alpha} W_{\alpha}+\text { (H.c. }\right)\right]-2 \kappa \int d^{4} \theta V .
\end{aligned}
$$

Here we have rescaled $V \rightarrow \frac{1}{g} V$. We have introduced the FI term which provides the D-term constraints on the component fields in $\Phi^{i}$. Note that for the Abelian gauge group,

\footnotetext{
${ }^{2}$ The case of $\Lambda=$ const. was studied in Appendix B of Ref. [49].
} 
we have $\quad \mathcal{D}_{\alpha} \Phi=D_{\alpha} \Phi+\left(D_{\alpha} V\right) \Phi, \quad \overline{\mathcal{D}}_{\dot{\alpha}} \Phi^{\dagger}=\bar{D}^{\dot{\alpha}} \Phi^{\dagger}+$ $\left(\bar{D}^{\dot{\alpha}} V\right) \Phi^{\dagger}$. The bosonic part of the Lagrangian (3.2) is then given by

$$
\begin{aligned}
\mathcal{L}_{\text {boson }}= & -D_{m} \varphi^{i} D^{m} \bar{\varphi}^{i}+F^{i} \bar{F}^{i}+D\left(\bar{\varphi}^{i} \varphi^{i}-\kappa\right) \\
& +\frac{1}{2 g} D^{2}-\frac{1}{4 g^{2}} F_{m n} F^{m n} \\
& +\delta_{i \bar{j}} \delta_{k \bar{l}} \Lambda(\varphi, \bar{\varphi})\left(\left(D_{m} \varphi^{i} D_{n} \bar{\varphi}^{\bar{j}}\right)\left(D^{m} \varphi^{k} D^{n} \bar{\varphi}^{\bar{l}}\right)\right. \\
& \left.-2\left(D_{m} \varphi^{i} D^{m} \bar{\varphi}^{\bar{j}}\right)\left(F^{k} \bar{F}^{\bar{l}}\right)+F^{i} \bar{F}^{\bar{j}} F^{k} \bar{F}^{\bar{l}}\right),
\end{aligned}
$$

where $F_{m n}=\partial_{m} A_{n}-\partial_{n} A_{m}$ and the gauge covariant derivative is defined by $D_{m} \varphi^{i}=\partial_{m} \varphi^{i}+i A_{m} \varphi^{i}$. In the following, we assume that the gauge invariant Kähler scalar $\Lambda(\varphi, \bar{\varphi})$ is given by a function of $X=D_{m} \varphi^{i} D^{m} \bar{\varphi}^{i}$ as a typical example. The most plausible reason for this assumption is that this quantity contains only the first order time derivative of fields. This guarantees the absence of the Ostrogradski's ghost [19] in the theory. This assumption is easily relaxed but allowing other gauge and Lorentz invariant quantities such as $X=\varphi^{i} \bar{\varphi}^{i}, D_{m} \varphi^{i} D^{n} \bar{\varphi}^{i} D^{m} \varphi^{j} D^{n} \bar{\varphi}^{j}$ and so on does not change the following discussions.

In the sigma model limit $g \rightarrow \infty$, the gauge kinetic and $D^{2}$ terms vanish and the gauge field $A_{m}$ becomes an auxiliary field. Before integrating out these fields, we first integrate out the auxiliary fields in the chiral superfields. The equation of motion for the auxiliary field $\bar{F}^{\bar{i}}$ is

$$
\begin{aligned}
0 & =\frac{\partial \mathcal{L}}{\partial \bar{F}^{\bar{i}}} \\
& =F^{i}-2 \Lambda(X)\left(\left(D_{m} \varphi^{j} D^{m} \bar{\varphi}^{j}\right) F^{i}-\left(F^{j} \bar{F}^{j}\right) F^{i}\right) .
\end{aligned}
$$

As we have noticed, there are two branches associated with the solutions $F^{i}=0$ and $F^{i} \neq 0$. We examine each branch separately.

\section{Canonical branch}

For the canonical branch corresponding to the solution $F^{i}=0$, the Lagrangian (3.3) becomes

$$
\begin{aligned}
\mathcal{L}_{\mathrm{c}}= & -D_{m} \varphi^{i} D^{m} \bar{\varphi}^{i}+\Lambda\left(D_{m} \varphi^{i} D_{n} \bar{\varphi}^{i}\right)\left(D^{m} \varphi^{j} D^{n} \bar{\varphi}^{j}\right) \\
& +D\left(\bar{\varphi}^{i} \varphi^{i}-\kappa\right) .
\end{aligned}
$$

The equation of motion for the auxiliary field $D$ gives the following constraint for the scalar fields $\varphi^{i}$ :

$$
\varphi^{i} \bar{\varphi}^{i}=\kappa
$$

The equation of motion for the gauge field $A_{m}$ is

$$
\begin{aligned}
0= & \frac{\partial \mathcal{L}}{\partial A_{m}} \\
= & -2 \kappa\left(i \kappa^{-1} \varphi^{i} \partial^{m} \bar{\varphi}^{i}+A^{m}\right)+i \frac{\partial \Lambda}{\partial X}\left(\varphi^{i} D^{m} \bar{\varphi}^{i}-\bar{\varphi}^{i} D^{m} \varphi^{i}\right) \\
& +2 i \Lambda(X)\left[\left(\varphi^{i} D_{p} \bar{\varphi}^{i}\right)\left(D_{m} \varphi^{j} D^{p} \bar{\varphi}^{j}\right)\right. \\
& \left.-\left(\bar{\varphi}^{i} D_{p} \varphi^{i}\right)\left(D_{m} \bar{\varphi}^{j} D^{p} \varphi^{j}\right)\right] .
\end{aligned}
$$

Using the constraint (3.6), we find that the solution to this equation is given by

$$
A_{m}=i \kappa^{-1} \bar{\varphi}^{i} \partial_{m} \varphi^{i}
$$

Substituting the solution (3.8) into (3.5) and using the constraint (3.6), we obtain the Lagrangian in the canonical branch,

$$
\mathcal{L}_{\mathrm{c}}=-\tilde{D}_{m} \bar{\varphi}^{i} \tilde{D}^{m} \varphi^{i}+\Lambda(\tilde{X})\left(\tilde{D}_{m} \varphi^{i} \tilde{D}_{n} \bar{\varphi}^{i}\right)\left(\tilde{D}^{m} \varphi^{j} \tilde{D}^{n} \bar{\varphi}^{j}\right),
$$

where we have defined $\tilde{D} \varphi^{i}=\partial_{m} \varphi^{i}-\kappa^{-1}\left(\bar{\varphi}^{j} \partial_{m} \varphi^{j}\right) \varphi^{i}$ and $\tilde{X}=\tilde{D}_{m} \varphi^{i} \tilde{D}^{m} \bar{\varphi}^{i}$.

It is convenient to solve the constraint (3.6) explicitly by using the parametrization

$$
\varphi^{i}=W^{i} \frac{\sqrt{\kappa}}{\sqrt{W^{\dagger} \cdot W}} .
$$

By using the $U(1)_{\mathbb{C}}$ gauge symmetry, we can fix $W^{1}=1$. Then, we have the conventional parametrization of the $\mathbb{C} P^{N-1}$ model,

$$
\begin{aligned}
\varphi^{i} & =\frac{\sqrt{\kappa}}{\sqrt{1+|\vec{u}|^{2}}}\left(\begin{array}{c}
1 \\
u^{s}
\end{array}\right), \\
\bar{\varphi}^{\bar{i}} & =\frac{\sqrt{\kappa}}{\sqrt{1+|\vec{u}|^{2}}}\left(1, \bar{u}^{\bar{s}}\right), \quad(s, \bar{s}=2, \ldots, N),
\end{aligned}
$$

where $u^{s}, \bar{u}^{\bar{s}}$ are inhomogeneous coordinates of the $\mathbb{C} P^{N-1}$ manifold. By using this form, the Lagrangian in the canonical branch can be rewritten as

$$
\begin{aligned}
\mathcal{L}_{\mathrm{c}}= & -\frac{\kappa}{\left(1+|\vec{u}|^{2}\right)^{2}}\left[\left(1+|\vec{u}|^{2}\right)\left(\partial_{m} \vec{u}^{*} \cdot \partial^{m} \vec{u}\right)-\left(\vec{u} \cdot \partial_{m} \vec{u}^{*}\right)\left(\vec{u}^{*} \cdot \partial^{m} \vec{u}\right)\right] \\
& +\frac{\kappa^{2}}{\left(1+|\vec{u}|^{2}\right)^{4}} \Lambda(u, \vec{u})\left[\left(1+|\vec{u}|^{2}\right)^{2}\left(\partial_{m} \vec{u}^{*} \cdot \partial_{n} \vec{u}\right)\left(\partial^{m} \vec{u}^{*} \cdot \partial^{n} \vec{u}\right)\right. \\
& \left.-2\left(1+|\vec{u}|^{2}\right)\left(\partial_{m} \vec{u}^{*} \cdot \partial_{n} \vec{u}\right)\left(\vec{u} \cdot \partial^{m} \vec{u}^{*}\right)\left(\vec{u}^{*} \cdot \partial^{n} \vec{u}\right)+\left(\vec{u} \cdot \partial_{m} \vec{u}^{*}\right)^{2}\left(\vec{u}^{*} \cdot \partial_{n} \vec{u}\right)^{2}\right] .
\end{aligned}
$$


By using the Fubini-Study metric

$$
g_{s \bar{t}}=\kappa \frac{\left(1+|\vec{u}|^{2}\right) \delta_{s \bar{t}}-u^{s} \bar{u}^{\bar{t}}}{\left(1+|\vec{u}|^{2}\right)^{2}}
$$

for the $\mathbb{C} P^{N-1}$ manifold, the Lagrangian (3.12) can be simply written as

$$
\begin{aligned}
\mathcal{L}_{\mathrm{c}}= & -g_{s \bar{t}} \partial_{m} u^{s} \partial^{m} \bar{u}^{\bar{t}} \\
& +g_{s \bar{t}} g_{q \bar{r}} \Lambda\left(u, \bar{u}, \partial_{m} u, \partial_{m} \bar{u}\right)\left(\partial_{m} u^{s} \partial_{n} \bar{u}^{\bar{t}}\right)\left(\partial^{m} u^{q} \partial^{n} \bar{u}^{\bar{r}}\right) .
\end{aligned}
$$

The first term is the ordinary kinetic term of the $\mathbb{C} P^{N-1}$ nonlinear sigma model and the second is the derivative corrections determined by the arbitrary scalar function $\Lambda\left(u, \bar{u}, \partial_{m} u, \partial_{m} \bar{u}\right)$.

Before going to the discussion on the noncanonical branch, two comments in this Lagrangian are in order. This Lagrangian with $\Lambda=$ const. was obtained in Ref. [49] as the low-energy effective theory of a BPS non-Abelian vortex in $\mathcal{N}=2$ supersymmetric $U(N)$ gauge theory [55]; a non-Abelian vortex in this case allows the supersymmetric $\mathbb{C} P^{N-1}$ model on the vortex world sheet, on which $1 / 2$ BPS condition preserves four supercharges among eight supercharges that the $\mathcal{N}=2$ supersymmetric theory in the bulk has. The bosonic part of the four-derivative correction term is precisely in this form [49].

Without supersymmetry, the $\mathbb{C} P^{N-1}$ Skyrme-Faddeev model was proposed in Refs. [46-48] as the $\mathbb{C} P^{N-1}$ model with four-derivative terms. In this case, there are three kinds of four-derivative terms, and the one in Eq. (3.14) (with $\Lambda=$ const.) corresponds to a particular choice among them. Thus, we call the Lagrangian in Eq. (3.14) (the bosonic part of) the supersymmetric $\mathbb{C} P^{N-1}$ SkyrmeFaddeev model.

\section{Noncanonical branch}

We next study the noncanonical branch corresponding to solutions $F_{i} \neq 0$. Again, the equation of motion for $\bar{F}$ is

$$
\begin{aligned}
0 & =\frac{\partial \mathcal{L}}{\partial \bar{F}^{\bar{i}}} \\
& =F^{i}-2 \Lambda(X)\left(\left(D_{m} \varphi^{j} D^{m} \bar{\varphi}^{j}\right) F^{i}-\left(F^{j} \bar{F}^{j}\right) F^{i}\right) .
\end{aligned}
$$

The solution $F^{i} \neq 0$ is found to be

$$
F^{i} \bar{F}^{i}=-\frac{1}{2 \Lambda}+D_{m} \varphi^{i} D^{m} \bar{\varphi}^{i}
$$

Substituting this solution into the Lagrangian (3.3), it becomes

$$
\begin{aligned}
\mathcal{L}_{\mathrm{nc}}= & \Lambda(X)\left[\left(D_{m} \bar{\varphi}^{i} D_{n} \varphi^{i}\right)\left(D^{m} \bar{\varphi}^{j} D^{n} \varphi^{j}\right)-\left(D_{m} \varphi^{j} D^{m} \bar{\varphi}^{j}\right)^{2}\right] \\
& -\frac{1}{4 \Lambda(X)}+D\left(\bar{\varphi}^{i} \varphi^{i}-\kappa\right)
\end{aligned}
$$

where we have assumed the sigma model limit $g \rightarrow \infty$. The D-term condition leads to the constraint (3.6), while the equation of motion for the gauge field is

$$
\begin{aligned}
0 & =i \frac{\partial \Lambda}{\partial X}\left(\varphi^{k} D^{m} \bar{\varphi}^{k}-\bar{\varphi}^{k} D^{m} \varphi^{k}\right)\left[\left(D_{m} \bar{\varphi}^{i} D_{n} \varphi^{i}\right)\left(D^{m} \bar{\varphi}^{j} D^{n} \varphi^{j}\right)\right. \\
& \left.-\left(D_{m} \varphi^{j} D^{m} \bar{\varphi}^{j}\right)^{2}\right] \\
& +2 i \Lambda(X)\left[-\bar{\varphi}^{j} D^{q} \varphi^{j}\left(D^{m} \bar{\varphi}^{i} D_{q} \varphi^{i}\right)+\varphi^{j} D^{p} \bar{\varphi}^{j}\left(D_{p} \bar{\varphi}^{i} D^{m} \varphi^{i}\right)\right. \\
& \left.-\left(D_{p} \varphi^{i} D^{p} \bar{\varphi}^{i}\right)\left(\varphi^{j} D^{m} \bar{\varphi}^{j}-\bar{\varphi}^{j} D^{m} \varphi^{j}\right)\right] \\
& +4 i \Lambda^{-2}(X) \frac{\partial \Lambda}{\partial X}\left(\varphi^{i} D^{m} \bar{\varphi}^{i}-\bar{\varphi}^{i} D^{m} \varphi^{i}\right) .
\end{aligned}
$$

By using the constraint (3.6), we find that Eq. (3.18) is again solved by

$$
A_{m}=i \kappa^{-1} \bar{\varphi}^{i} \partial_{m} \varphi^{i}
$$

Substituting this solution into the Lagrangian (3.17), we find the new sigma model given by

$$
\begin{aligned}
\mathcal{L}_{\mathrm{nc}}= & \Lambda(\tilde{X})\left[\left(\tilde{D}_{m} \bar{\varphi}^{i} \tilde{D}_{n} \varphi^{i}\right)\left(\tilde{D}^{m} \bar{\varphi}^{j} \tilde{D}^{n} \varphi^{j}\right)-\left(\tilde{D}_{m} \varphi^{j} \tilde{D}^{m} \bar{\varphi}^{j}\right)^{2}\right] \\
& -\frac{1}{4 \Lambda(\tilde{X})}
\end{aligned}
$$

The expression (3.11) enables us to rewrite the Lagrangian (3.20) as

$$
\begin{aligned}
\mathcal{L}_{\mathrm{nc}}= & \frac{\kappa^{2}}{\left(1+|\vec{u}|^{2}\right)^{4}} \Lambda\left(u, \vec{u}, \partial_{m} u, \partial_{m} \bar{u}\right)\left[\left(1+|\vec{u}|^{2}\right)^{2}\left\{\left(\partial_{m} \vec{u} \cdot \partial_{n} \vec{u}^{*}\right)\left(\partial^{m} \vec{u} \cdot \partial^{n} \vec{u}^{*}\right)-\left(\partial_{m} \vec{u}^{*} \cdot \partial^{m} \vec{u}\right)^{2}\right\}\right. \\
& -2\left(1+|\vec{u}|^{2}\right)\left\{\left(\partial_{m} \vec{u}^{*} \cdot \partial_{n} \vec{u}\right)\left(\vec{u} \cdot \partial^{m} \vec{u}^{*}\right)\left(\vec{u}^{*} \cdot \partial^{n} \vec{u}\right)-\left(\partial_{m} \vec{u}^{*} \cdot \partial^{m} \vec{u}\right)\left(\vec{u} \cdot \partial_{n} \vec{u}^{*}\right)\left(\vec{u}^{*} \cdot \partial^{n} \vec{u}\right)\right\} \\
& \left.+\left(\vec{u} \cdot \partial_{m} \vec{u}^{*}\right)^{2}\left(\vec{u}^{*} \cdot \partial_{n} \vec{u}\right)^{2}-\left\{\left(\vec{u} \cdot \partial_{m} \vec{u}^{*}\right)\left(\vec{u}^{*} \cdot \partial^{m} \vec{u}\right)\right\}^{2}\right]-\frac{1}{4} \Lambda^{-1}\left(u, \vec{u}, \partial_{m} u, \partial_{m} \vec{u}\right) .
\end{aligned}
$$


By using the Fubini-Study metric (3.13), this can be simply expressed as

$$
\begin{aligned}
\mathcal{L}_{\mathrm{nc}}= & \Lambda\left(u, \bar{u}, \partial_{m} u, \partial_{m} \bar{u}\right) \\
& \times\left[\left(g_{s \bar{t}} \partial_{m} u^{s} \partial_{n} \bar{u}^{\bar{t}}\right)\left(g_{q \bar{r}} \partial^{m} u^{q} \partial^{n} \bar{u}^{\bar{q}}\right)-\left(g_{s \bar{t}} \partial_{m} u^{s} \partial^{m} \bar{u}^{\bar{t}}\right)^{2}\right] \\
& -\frac{1}{4} \Lambda^{-1}\left(u, \bar{u}, \partial_{m} u, \partial_{m} \bar{u}\right) .
\end{aligned}
$$

This four-derivative term does not contain fourth order time derivatives, unlike the one in Eq. (3.14) in the canonical branch. It is obvious that the target space of the scalar fields is the $\mathbb{C} P^{N-1}$ space, but the canonical kinetic term $g_{s \bar{t}} \partial_{m} u^{s} \partial^{m} u^{\bar{t}}$ is absent.

In particular, for the $N=2$ case of $\mathbb{C} P^{1}$, the Lagrangian reduces to

$$
\mathcal{L}_{\mathrm{nc}}=-\kappa^{2} \Lambda \frac{\left(\partial_{m} u \partial^{m} \bar{u}\right)^{2}-\left|\partial_{m} u \partial^{m} u\right|^{2}}{\left(1+|u|^{2}\right)^{4}}-\frac{1}{4 \Lambda},
$$

which is known as the supersymmetric baby Skyrme model $[24,25,27,40-42]$. One notices that the first term (with $\Lambda=1$ ) is nothing but the Skyrme-Faddeev fourth derivative term (without the fourth order time derivatives), while the second term provides higher derivative corrections determined by $\Lambda\left(u, \bar{u}, \partial_{m} u, \partial_{m} \bar{u}\right)$. The Lagrangian in Eq. (3.22) may be called the supersymmetric $\mathbb{C} P^{N-1}$ baby Skyrme model.

\section{B. $G_{M, N}$ model}

We next consider the nonlinear sigma model whose target space is the Grassmann manifold $G_{M, N}=$ $U(M) /[U(M-N) \times U(N)]$. We consider a gauged linear sigma model with the global symmetry $G=S U(M)$ and gauge symmetry $U(N)$, of which the chiral superfield $\Phi_{a}^{i}$ $(i=1, \ldots M ; a=1, \ldots N)$ belong to the $(\mathbf{M}, \overline{\mathbf{N}})$ representation. The global symmetry $G=S U(M)$ and gauge symmetry $U(N)_{\mathbb{C}}=G L(N, \mathbb{C})$ act on it as

$$
\Phi \rightarrow g \Phi e^{i \Theta^{\prime}}, \quad e^{2 V} \rightarrow e^{-i \Theta^{\prime}} e^{2 V} e^{i \Theta^{\prime \dagger}},
$$

where $g \in S U(M), \Theta^{\prime}(x, \theta, \bar{\theta})$ is a chiral superfield of a $U(N)_{\mathbb{C}}$ gauge parameter, and we have introduced the associated $U(N)$ vector superfield $V(x, \theta, \bar{\theta})$. The $G \times G^{\prime}$ invariant Lagrangian is therefore [52,54]

$$
\begin{aligned}
\mathcal{L} & =\int d^{4} \theta \operatorname{Tr}\left[\Phi e^{2 V} \Phi^{\dagger}\right] \\
& -\frac{1}{16} \int d^{4} \theta \Lambda\left(\Phi, \Phi^{\dagger}\right) \operatorname{Tr}\left[\mathcal{D}^{\alpha} \Phi e^{2 V} \overline{\mathcal{D}}_{\dot{\alpha}} \Phi^{\dagger}\right] \operatorname{Tr}\left[\mathcal{D}_{\alpha} \Phi e^{2 V} \overline{\mathcal{D}}^{\dot{\alpha}} \Phi^{\dagger}\right] \\
& +\frac{1}{16 g^{2}}\left(\int d^{2} \theta \operatorname{Tr}\left[W^{\alpha} W_{\alpha}\right]+(\text { H.c. })\right)-2 \kappa \int d^{4} \theta \operatorname{Tr} V .
\end{aligned}
$$

The derivative corrections are given in the double trace form, and we have included the FI term which provides the D-term conditions on the chiral multiplets.

In the nonlinear sigma model limit $g \rightarrow \infty$, the bosonic part of the Lagrangian becomes

$$
\begin{aligned}
\mathcal{L}_{\text {boson }}= & -\operatorname{Tr}\left[D_{m} \varphi D^{m} \bar{\varphi}\right]+\operatorname{Tr}[\bar{\varphi} D \varphi-\kappa D]+\operatorname{Tr}[F \bar{F}] \\
& +\Lambda(\varphi, \bar{\varphi})\left\{\operatorname{Tr}\left[D_{m} \varphi D_{n} \bar{\varphi}\right] \operatorname{Tr}\left[D^{m} \varphi D^{n} \bar{\varphi}\right]\right. \\
& -\operatorname{Tr}\left[D_{m} \varphi D^{m} \bar{\varphi}\right] \operatorname{Tr}[F \bar{F}] \\
& \left.-\operatorname{Tr}\left[F D_{m} \bar{\varphi}\right] \operatorname{Tr}\left[D^{m} \varphi \bar{F}\right]+\operatorname{Tr}[F \bar{F}] \operatorname{Tr}[F \bar{F}]\right\}
\end{aligned}
$$

The gauge covariant derivative is $D_{m} \varphi=\partial_{m} \varphi+i \varphi A_{m}$. In the following, we assume $\Lambda$ is given by the gauge invariant quantity $X=\operatorname{Tr}\left[D_{m} \varphi D^{m} \bar{\varphi}\right]$. As clarified before, this condition is easily relaxed allowing for any other gauge invariant quantities. The equation of motion for the auxiliary field $D$ gives the constraint

$$
\bar{\varphi} \varphi=\kappa \mathbf{1}_{N} .
$$

On the other hand, the equation of motion for the auxiliary field $\bar{F}$ is given by

$$
\begin{aligned}
& F+\Lambda(X)\left\{-\operatorname{Tr}\left[D_{m} \varphi D^{m} \bar{\varphi}\right] F\right. \\
& \left.\quad-\operatorname{Tr}\left[F D_{m} \bar{\varphi}\right] D^{m} \varphi+2 \operatorname{Tr}[F \bar{F}] F\right\}=0 .
\end{aligned}
$$

\section{Canonical branch}

It is obvious that $F=0$ is a solution. In this case, the Lagrangian becomes

$$
\mathcal{L}_{\mathrm{c}}=-\operatorname{Tr}\left[D_{m} \varphi D^{m} \bar{\varphi}\right]+\Lambda(X) \operatorname{Tr}\left[D_{m} \varphi D_{n} \bar{\varphi}\right] \operatorname{Tr}\left[D^{m} \varphi D^{n} \bar{\varphi}\right]
$$

where the scalar fields satisfy the constraint (3.27). The equation of motion for $A_{m}$ then becomes

$$
\begin{aligned}
& \bar{\varphi} \partial_{m} \varphi+i \kappa A_{m}+i \frac{\partial \Lambda}{\partial X}\left(\varphi D^{m} \bar{\varphi}-D^{m} \varphi \bar{\varphi}\right) \\
& \quad-\Lambda(X) \operatorname{Tr}\left[D_{m} \varphi D_{n} \bar{\varphi}+D_{n} \varphi D_{m} \bar{\varphi}\right]\left(\bar{\varphi} \partial^{n} \varphi+i \kappa A^{n}\right)=0
\end{aligned}
$$

where we have used the constraint (3.27). We again find that the exact solution to this equation is given by

$$
A_{m}=i \kappa^{-1} \bar{\varphi} \partial_{m} \varphi
$$

Plugging this solution back into the Lagrangian, we obtain the nonlinear sigma model supplemented by the derivative corrections 
$\mathcal{L}_{\mathrm{c}}=-\operatorname{Tr}\left[\tilde{D}_{m} \varphi \tilde{D}^{m} \bar{\varphi}\right]+\Lambda(\tilde{X}) \operatorname{Tr}\left[\tilde{D}_{m} \varphi \tilde{D}_{n} \bar{\varphi}\right] \operatorname{Tr}\left[\tilde{D}^{m} \varphi \tilde{D}^{n} \bar{\varphi}\right]$.

Here $\tilde{D}_{m} \varphi=\partial_{m} \varphi-\kappa^{-1} \varphi\left(\bar{\varphi} \partial_{m} \varphi\right)$ and $\tilde{X}=\operatorname{Tr}\left[\tilde{D}_{m} \varphi \tilde{D}^{m} \bar{\varphi}\right]$. The constraint (3.27) is solved by the parametrization

$$
\varphi=W \frac{\sqrt{\kappa}}{\sqrt{W^{\dagger} W}},
$$

where $W$ is an $M \times N$ matrix. Substituting this into the Lagrangian, the first term in (3.32) becomes the kinetic term of the sigma model whose target space is the Grassmann manifold $G_{M, N}$, while the second term gives derivative corrections determined by the arbitrary gauge invariant function $\Lambda\left(W, W^{\dagger}, \partial_{m} W, \partial_{m} W^{\dagger}\right)$.

\section{Noncanonical branch}

We next examine the noncanonical branch. It is obvious that there are nonzero solutions $F \neq 0$ to Eq. (3.28). Compared with the case of the Abelian gauge symmetry, it is not straightforward to write down explicit solutions for $F$. However, the gauge invariant solution $\operatorname{Tr}[F \bar{F}]$ should be given by the gauge and the Lorentz invariant quantities $Y$ such as

$$
Y=\operatorname{Tr}\left[D_{m} \varphi D^{m} \bar{\varphi}\right], \quad \operatorname{Tr}\left[D_{m} \varphi D_{n} \bar{\varphi} D^{m} \varphi D^{n} \bar{\varphi}\right],
$$

$$
\operatorname{Tr}\left[D_{m} \varphi D_{n} \bar{\varphi} D^{n} \varphi D^{m} \bar{\varphi}\right],
$$

and so on. Using the equation of motion for $F$, the noncanonical Lagrangian becomes

$$
\begin{aligned}
\mathcal{L}_{\mathrm{nc}}= & -\operatorname{Tr}\left[D_{m} \varphi D^{m} \bar{\varphi}\right] \\
& +\Lambda(X)\left\{\operatorname{Tr}\left[D_{m} \varphi D_{n} \bar{\varphi}\right] \operatorname{Tr}\left[D^{m} \varphi D^{n} \bar{\varphi}\right]-\operatorname{Tr}[F \bar{F}(Y)]^{2}\right\} .
\end{aligned}
$$

Here $\operatorname{Tr}[F \bar{F}(Y)]$ is a solution $F \neq 0$ given by $Y$. The variation of the Lagrangian (3.35) by $A_{m}$ is therefore

$$
\begin{aligned}
\delta \mathcal{L}_{\mathrm{nc}}= & i \operatorname{Tr}\left[\left(\bar{\varphi} \partial^{m} \varphi+i \kappa A^{m}\right) \delta A_{m}\right] \\
& +i \frac{\partial \Lambda}{\partial X} \operatorname{Tr}\left[\left(\varphi D^{m} \bar{\varphi}-D^{m} \varphi \bar{\varphi}\right) \delta A_{m}\right] \\
& -i \Lambda(X) \operatorname{Tr}\left[D^{m} \varphi D^{n} \bar{\varphi}+D^{n} \varphi D^{m} \bar{\varphi}\right] \\
& \times \operatorname{Tr}\left[\left(\bar{\varphi} \partial_{n} \varphi+i \kappa A_{n}\right) \delta A_{m}\right] \\
& -2 \Lambda(\varphi, \bar{\varphi}) \operatorname{Tr}[F \bar{F}] \operatorname{Tr}\left[(F \bar{F})^{\prime} \delta Y\right] .
\end{aligned}
$$

Here $(F \bar{F})^{\prime}$ stands for the differentiation with respect to $Y$. In the last term in (3.36), one finds that the variation of the gauge invariant quantity $Y$ is proportional to $\bar{\varphi} \partial^{m} \varphi+i \kappa A^{m}$. Indeed, for $Y=\operatorname{Tr}\left[D_{m} \varphi D^{m} \bar{\varphi}\right]$, we have

$$
\delta Y=2 i \operatorname{Tr}\left[\left(\bar{\varphi} \partial^{m} \varphi+i \kappa A^{m}\right) \delta A_{m}\right] .
$$

Therefore, even in the noncanonical branch, we find that the solution to the equation of motion for $A_{m}$ is given by $A_{m}=i \kappa^{-1} \varphi \partial_{m} \bar{\varphi}$. For the other gauge invariant combinations $Y$ such as (3.34), the result is the same. The constraint (3.27) is solved by the parametrization (3.33) and the Lagrangian becomes again the Grassmanian sigma model with the derivative corrections.

We comment on the symmetric nature of the Grassmanian manifold $G_{M, N}=G_{M, M-N}$. We have constructed the Grassmanian sigma models by gauging the symmetry $G^{\prime}=U(N)$, but there is an alternative way to define the same target space by gauging $G^{\prime}=U(M-N)$. It is obvious that the latter construction is essentially the same with the former one except the $N=1$ case. In this case, one notices that $G_{N, 1}=G_{N, N-1}=\mathbb{C} P^{N-1}$. This equality implies that we can construct supersymmetric sigma model whose target space is $\mathbb{C} P^{N-1}$, either by gauging the non-Abelian $U(N-1)$ symmetry or the Abelian $U(1)$ symmetry. The former is nothing but the construction discussed in this section, while the latter is the formulation discussed in the previous section. We find apparently different Lagrangians (3.35) and (3.20) in the derivative corrections. This means that the equivalence of the target spaces in different formalism are not taken over to the derivative corrections in the superfield language. In the next section, we will see a similar situation in the canonical branch of the nonlinear sigma models defined by the F-term constraints. We will, among other things, encounter qualitatively different structures of the target spaces in the noncanonical branch.

\section{NONLINEAR SIGMA MODELS WITH D- AND F-TERM CONSTRAINTS}

In this section, we discuss the nonlinear sigma models defined by F-term constraints in addition to the D-term constraints [52,56]. Such Hermitian symmetric target spaces include the quadric surface $Q^{N-2}=$ $S O(N) /[S O(N-2) \times U(1)]$, the quotient spaces of the types $S O(2 N) / U(N), S p(2 N) / U(N), E_{6} /[S O(10) \times U(1)]$, and $E_{7} /\left[E_{6} \times U(1)\right]$. We begin with the target space $Q^{N-2}=$ $S O(N) /[S O(N-2) \times U(1)]$ as a typical example.

\section{A. Quadric surface $Q^{N-2}$}

We consider a gauged linear sigma model with the global symmetry $G=S O(N)$ and the gauge symmetry $U(1)$. The chiral superfield $\Phi^{i}(i=1, \ldots, N)$ belongs to the fundamental representation $\mathbf{N}$ of $S O(N)$ and has the $U(1)$ charge +1 . The Lagrangian in the nonlinear sigma model limit is

$$
\begin{aligned}
\mathcal{L}= & \int d^{4} \theta\left(\Phi^{i} e^{2 V} \Phi^{\dagger i}-2 \kappa V\right)+\left(\int d^{2} \theta \Phi_{0} \vec{\Phi}^{t} J \vec{\Phi}+\text { H.c. }\right) \\
& +\frac{1}{16} \int d^{4} \theta \Lambda\left(\Phi, \Phi^{\dagger}\right) e^{4 V} \mathcal{D}^{\alpha} \Phi^{i} \mathcal{D}_{\alpha} \Phi^{j} \overline{\mathcal{D}}_{\dot{\alpha}} \Phi^{\dagger i} \overline{\mathcal{D}}^{\dot{\alpha}} \Phi^{\dagger j},
\end{aligned}
$$


where we have introduced the gauge invariant superpotential $W=\Phi_{0} \vec{\Phi}^{t} J \vec{\Phi}$. This will provide the F-term constraints on the chiral multiplet. Here $\Phi_{0}$ is the $S O(N)$ singlet and whose $U(1)$ charge is assigned to $-2, V$ is the $U(1)$ vector multiplet, and the matrix $J$ is given by

$$
J=\left(\begin{array}{ccc}
0 & 0 & 1 \\
0 & \mathbf{1}_{N-2} & 0 \\
1 & 0 & 0
\end{array}\right) .
$$

The gauge transformations are given by

$$
\Phi \rightarrow e^{-i \Phi^{\prime}} \Phi, \quad \Phi_{0} \rightarrow e^{2 i \Theta^{\prime}} \Phi_{0}, \quad e^{2 V} \rightarrow e^{-i \Theta^{\prime \dagger}} e^{2 V} e^{i \Theta^{\prime}} .
$$

Then the bosonic part of the Lagrangian is evaluated as

$$
\begin{aligned}
\mathcal{L}_{\text {boson }}= & -D_{m} \varphi^{i} D^{m} \bar{\varphi}^{i}+F^{i} \bar{F}^{i}+F_{0}\left(\vec{\varphi}^{t} J \vec{\varphi}\right)+\bar{F}_{0}\left(\overrightarrow{\vec{\varphi}}^{t} J \overrightarrow{\bar{\varphi}}\right)+D\left(\bar{\varphi}^{i} \varphi^{i}-\kappa\right) \\
& +\Lambda(X)\left\{\left(D_{m} \varphi^{i} D_{n} \bar{\varphi}^{i}\right)\left(D^{m} \varphi^{j} D^{n} \bar{\varphi}^{j}\right)-2\left(D_{m} \varphi^{i} D^{m} \bar{\varphi}^{i}\right) F^{j} \bar{F}^{j}+\left(F^{i} F^{i}\right)^{2}\right\} \\
& +2 \varphi_{0}\left\{\varphi^{1} F^{N}+\varphi^{N} F^{1}+\sum_{\hat{i}=2}^{N-1} \varphi^{\hat{i}} F^{\hat{i}}\right\}+2 \bar{\varphi}_{0}\left\{\bar{\varphi}^{1} \bar{F}^{N}+\bar{\varphi}^{N} \bar{F}^{1}+\sum_{\hat{i}=2}^{N-1} \bar{\varphi}^{\hat{i}} \bar{F}^{\hat{i}}\right\},
\end{aligned}
$$

where we have again assumed that $\Lambda$ is a function of the gauge invariant quantity $X=D_{m} \varphi^{i} D^{m} \bar{\varphi}^{i}$. The equation of motion for $D$ gives the constraint

$$
\bar{\varphi}^{i} \varphi^{i}=\kappa
$$

Since $\Phi_{0}$ does not propagate, it is also integrated out. The F-term constraints are given by the equation of motions for $F_{0}, \varphi_{0}$,

$$
\begin{gathered}
\delta F_{0}: \vec{\varphi}^{t} J \vec{\varphi}=0, \\
\delta \varphi_{0}: \varphi^{1} F^{N}+\varphi^{N} F^{1}+\sum_{\hat{i}=2}^{N-1} \varphi^{\hat{i}} F^{\hat{i}}=0 .
\end{gathered}
$$

These give the constraints for the fields $\varphi^{i}, \bar{\varphi}^{i}$. The equations of motion for $\bar{F}^{i}$ are

$$
\begin{aligned}
\delta \bar{F}^{1}: F^{1}-2 \Lambda(X)\left\{\left(D_{m} \varphi^{j} D^{m} \bar{\varphi}^{j}\right) F^{1}-\left(F^{j} \bar{F}^{j}\right) F^{1}\right\} \\
\quad+2 \bar{\varphi}_{0} \bar{\varphi}^{N}=0, \\
\delta \bar{F}^{\hat{i}}: F^{\hat{i}}-2 \Lambda(X)\left\{\left(D_{m} \varphi^{j} D^{m} \bar{\varphi}^{j}\right) F^{\hat{i}}-\left(F^{j} \bar{F}^{j}\right) F^{\hat{i}}\right\} \\
\quad+2 \bar{\varphi}_{0} \bar{\varphi}^{\hat{i}}=0,(\hat{i}=2, \ldots, N-1), \\
\delta \bar{F}^{N}: F^{N}-2 \Lambda(X)\left\{\left(D_{m} \varphi^{j} D^{m} \bar{\varphi}^{j}\right) F^{N}-\left(F^{j} \bar{F}^{j}\right) F^{N}\right\} \\
+2 \bar{\varphi}_{0} \bar{\varphi}^{1}=0 .
\end{aligned}
$$

Equations (4.5)-(4.8) should be solved simultaneously. In the following, we solve these equations in the canonical and the noncanonical branches separately.

\section{Canonical branch}

One finds that a solution to Eq. (4.8) is given by

$$
\varphi_{0}=0, \quad F^{i}=0 .
$$

This corresponds to the canonical branch. We note that in this branch, the constraint (4.7) becomes trivial. Then the Lagrangian becomes

$$
\mathcal{L}_{\mathrm{c}}=-D_{m} \varphi^{i} D^{m} \bar{\varphi}^{i}+\Lambda(X)\left(D_{m} \varphi^{i} D_{n} \bar{\varphi}^{i}\right)\left(D^{m} \varphi^{j} D^{n} \bar{\varphi}^{j}\right) .
$$

The equation of motion for $A_{m}$ is therefore

$$
\begin{aligned}
- & \kappa\left(i \kappa^{-1} \varphi^{i} \partial_{m} \bar{\varphi}^{i}+A_{m}\right)+i \frac{\partial \Lambda}{\partial X}\left(\varphi^{i} D^{m} \bar{\varphi}^{i}-\bar{\varphi}^{i} D^{m} \varphi^{i}\right) \\
& +i \Lambda(\varphi, \bar{\varphi})\left\{\left(\varphi^{i} D_{n} \bar{\varphi}^{i}\right)\left(D_{m} \varphi^{j} D^{n} \bar{\varphi}^{j}\right)\right. \\
& \left.-\left(\bar{\varphi}^{i} D^{n} \varphi^{i}\right)\left(D_{m} \bar{\varphi}^{j} D^{n} \varphi^{j}\right)\right\}=0,
\end{aligned}
$$

where we have used the constraint (4.5). We find that the solution is given by

$$
A_{m}=i \kappa^{-1} \bar{\varphi}^{i} \partial_{m} \varphi^{i}
$$

Substituting this solution to the Lagrangian, the covariant derivative $D_{m} \varphi^{i}$ in the Lagrangian (4.10) is replaced by $\tilde{D}_{m} \varphi^{i}$. Now, we solve the remaining constraints (4.5) and (4.6). The constraint (4.5) is solved by the following parametrization:

$$
\varphi^{i}=W^{i} \frac{\sqrt{\kappa}}{\sqrt{W^{\dagger} \cdot W}} .
$$

Since we have 


$$
\begin{gathered}
\vec{\varphi}^{t} J \vec{\varphi}=\frac{\kappa}{W^{\dagger} \cdot W}\left(2 W^{1} W^{N}+\left(W^{\hat{i}}\right)^{2}\right)=0, \\
(\hat{i}=2, \ldots, N-1),
\end{gathered}
$$

the last constraint (4.6) is solved by

$$
W^{i}=\left(\begin{array}{c}
1 \\
u^{\hat{i}} \\
-\frac{1}{2}\left(u^{\hat{i}}\right)^{2}
\end{array}\right)
$$

where we have fixed $W^{1}=1$ by the $U(1)_{\mathbb{C}}$ gauge transformation. Plugging this back into the Lagrangian, we obtain the $Q^{N-2}$ sigma model whose derivative corrections are completely determined by $\Lambda\left(W, W^{\dagger}, \partial_{m} W, \partial_{m} W^{\dagger}\right)$.

\section{Noncanonical branch}

In the noncanonical branch, we find that a solution to the equations (4.8) is given by

$$
\varphi_{0}=0, \quad F^{i} \bar{F}^{i}=-\frac{1}{2 \Lambda(X)}+D_{m} \varphi^{i} D^{m} \bar{\varphi}^{i} .
$$

Then, the Lagrangian becomes

$$
\begin{aligned}
\mathcal{L}_{\mathrm{nc}}= & \Lambda(X)\left[\left(D_{m} \bar{\varphi}^{i} D_{n} \varphi^{i}\right)\left(D^{m} \bar{\varphi}^{j} D^{n} \varphi^{j}\right)-\left(D_{m} \varphi^{j} D^{m} \bar{\varphi}^{j}\right)^{2}\right] \\
& -\frac{1}{4 \Lambda(X)} .
\end{aligned}
$$

The equation of motion for $A_{m}$ is the same with (3.18) and it is again solved by (3.19). Substituting this into the Lagrangian, the gauge covariant derivative $D_{m} \varphi^{i}$ is replaced by $\tilde{D}_{m} \varphi^{i}$. The remaining constraints (4.5) and (4.6) are solved by the parametrizations (4.13) and (4.15) and the target space is $Q^{N-2}$. However, we should keep in mind that there is an extra constraint (4.7) given by

$$
\varphi^{1} F^{N}(\tilde{X})+\varphi^{N} F^{1}(\tilde{X})+\sum_{\hat{i}=2}^{N-1} \varphi^{\hat{i}} F^{\hat{i}}(\tilde{X})=0,
$$

where $F^{i}(\tilde{X}) \neq 0$ is the solution to the auxiliary field in the noncanonical branch. The constraint (4.18) generically contains space-time derivatives of the fields $\varphi^{i}, \bar{\varphi}^{\bar{i}}$ and this has to be solved together with the following equation of motion:

$$
\begin{aligned}
& \Lambda^{\prime}(\tilde{X}) \tilde{D}^{m} \varphi^{i}\left[\left(\tilde{D}_{m} \bar{\varphi}^{j} \tilde{D}_{n} \varphi^{j}\right)\left(\tilde{D}^{m} \bar{\varphi}^{k} \tilde{D}^{n} \varphi^{k}\right)-\left(\tilde{D}_{m} \varphi^{j} \tilde{D}^{m} \bar{\varphi}^{j}\right)^{2}\right] \\
& \quad-2 \Lambda(\tilde{X})\left[\tilde{D}_{n} \varphi^{i}\left(\tilde{D}^{m} \bar{\varphi}^{j} \tilde{D}^{n} \varphi^{j}\right)-\tilde{D}_{m} \varphi^{i}\left(\tilde{D}_{n} \varphi^{j} \tilde{D}^{n} \bar{\varphi}^{j}\right)\right] \\
& \quad+\frac{1}{4 \Lambda^{2}(\tilde{X})} \Lambda^{\prime}(\tilde{X}) \tilde{D}^{m} \varphi^{i}=0
\end{aligned}
$$

where the prime in $\Lambda^{\prime}(\tilde{X})$ denotes the differentiation with respect to $\tilde{X}$. In general, it is uncertain whether the simultaneous equations (4.18) and (4.19) admit nontrivial solutions or not. Instead, the most plausible way to work with these equations is to restrict the dynamics to a subspace in $Q^{N-2}$. This subspace highly depends on explicit structures of the solution $F^{i}$. For example, if we consider the $N=4$ case, one finds that a solution to the equation of motion for $F^{i}$ in the noncanonical branch is

$F^{2}=F^{3}=F^{4}=0, \quad F^{1}=\sqrt{-\frac{1}{2 \Lambda(\tilde{X})}+\tilde{D}_{m} \varphi^{i} \tilde{D}^{m} \bar{\varphi}^{i}}$.

Substituting this solution to the constraint (4.18) gives a condition

$$
\varphi^{4} \sqrt{-\frac{1}{2 \Lambda(\tilde{X})}+\tilde{D}_{m} \varphi^{i} \tilde{D}^{m} \bar{\varphi}^{i}}=0 .
$$

In order that this is compatible with equation of motion, we first choose the subspace $\varphi^{4}=0$ in $Q^{2}$ and then solve Eq. (4.19) in that subspace.

A comment is in order. As for the Grassmann case, we have an equality $Q^{4}=G_{4,2}$. The constraint (4.18) is necessary in the construction of the $Q^{4}$ sigma model discussed in this section, but such a restriction to a subspace was absent in the construction of the $G_{4,2}$ model in the previous section. Apparently, they have different Lagrangians even though there is a target space isomorphism between them. The same is true for the relation $Q^{1}=G_{2,1}=\mathbb{C} P^{1}$. In the noncanonical branch, the different constructions of sigma models result in the different derivative corrections and the field space that the dynamics occurs, although they give identical models in the canonical branches.

\section{B. $S O(2 N) / U(N)$ and $S p(N) / U(N)$ models}

Observed that the $Q^{N-2}$ model has remarkable structures in the noncanonical branch due to the F-term constraint, we next consider nonlinear sigma models with the target spaces $S O(2 N) / U(N)$ or $S p(N) / U(N)$. The gauged linear sigma model has the global symmetry $G=S O(2 N)$ or $S p(N)[=U S p(2 N)]$ and gauge symmetry $G^{\prime}=U(N)$. The chiral superfield $\Phi$ belongs to the $(\mathbf{2 N}, \overline{\mathbf{N}})$ representation of $G \times G^{\prime}$ and it is expressed as an $2 N \times N$ matrix. The constraint is given by the F-term superpotential

$$
W=\operatorname{Tr}\left[\Phi_{0} \Phi^{t} J^{\prime} \Phi\right],
$$

where $\Phi_{0}$ is an $N \times N$ symmetric (antisymmetric) matrix superfield satisfying $\Phi_{0}^{t}=\epsilon \Phi_{0}$ for $G=S O(2 N)[S p(N)]$. Here $\epsilon=1$ for $G=S O(2 N)$ and $\epsilon=-1$ for $G=S p(N)$. The $U(1)_{D} \subset U(N)$ charge of $\Phi_{0}$ is assigned to -2 to cancel the $U(1)$ charge +1 of $\Phi$. The $2 N \times 2 N$ matrix $J^{\prime}$ is given by 


$$
J^{\prime}=\left(\begin{array}{cc}
0 & \mathbf{1}_{N} \\
\epsilon \mathbf{1}_{N} & 0
\end{array}\right)
$$

which is an invariant tensor of $\operatorname{SO}(2 N)(\epsilon=1)$ or $\operatorname{Sp}(N)(\epsilon=-1)$.

The equation of motion for $D$ gives the constraint (3.27), while that for $F_{0}$ gives the constraint

$$
\varphi^{t} J^{\prime} \varphi=0
$$

and that for $\varphi_{0}$ gives a constraint

$$
\varphi^{t} J^{\prime} F=0
$$

The equation for $F$ is (4.8), but the gauge symmetry is nonAbelian. One finds that $\varphi_{0}=0$ and $F=0$ are a solution to the equation of motion for $F$. Therefore, the constraint (4.25) becomes trivial in the canonical branch. We find that the gauge field is integrated out by the solution $A_{m}=$ $i \kappa^{-1} \bar{\varphi} \partial_{m} \varphi$ in the sigma model limit which is the same with the case without the derivative corrections. Then the target space of the sigma model is nothing but $S O(2 N) / U(N)$ or $S p(N) / U(N)$ and the derivative corrections appear as in the Grassmann case in Eq. (3.35).

Compared with the $Q^{N-2}$ model, it is not straightforward to write down solutions $F \neq 0$ explicitly due to the non-Abelian gauge symmetry. However, similar to the Grassmann case, the solution $\operatorname{Tr}[F \bar{F}]$ is given by the gauge invariant quantities such as $\operatorname{Tr}\left[D_{m} \varphi D^{m} \bar{\varphi}\right]$. Therefore, we again find that the gauge field $A_{m}$ is integrated out by $A_{m}=i \kappa^{-1} \bar{\varphi} \partial_{m} \varphi$. The subsequent discussion is parallel to the $Q^{N-2}$ case. Due to the extra constraint (4.25), we find that the target spaces $S O(2 N) / U(N)$ or $S p(N) / U(N)$ should be restricted to their subspaces in the noncanonical branches.

\section{C. $E_{6} /[S O(10) \times U(1)]$ and $E_{7} /\left[E_{6} \times U(1)\right]$ models}

For the case of the target space $E_{6} /[S O(10) \times U(1)]$, the global symmetry is $G=E_{6}$ and the $G^{\prime}=U(1)$ symmetry is gauged in the gauged linear sigma model [52]. The chiral superfield $\Phi$ belongs to the fundamental representation 27 of $E_{6}$. This is decomposed into the maximal subgroup $S O(10) \times U(1)$. The F-term constraint is given by the superpotential

$$
W=\Gamma_{i j k} \Phi_{0}^{i} \Phi^{j} \Phi^{k},
$$

where $\Gamma_{i j k}$ is a rank-3 symmetric invariant tensor of $E_{6}$. Since the gauge symmetry is Abelian, the structure of the sigma model is essentially the same with the $Q^{N-2}$ case. Due to the constraint

$$
\Gamma_{i j k} \varphi^{i} F^{k}=0
$$

derived by the superpotential, the target space of the sigma model is restricted to a subspace of $E_{6} /[S O(10) \times U(1)]$ in the noncanonical branch.

For the target space $E_{7} /\left[E_{6} \times U(1)\right]$, the global symmetry is $G=E_{7}$ and the $G^{\prime}=U(1)$ symmetry is gauged in the gauge linear sigma model. The F-term constraint is [52]

$$
W=d_{\alpha \beta \gamma \delta} \Phi_{0}^{\alpha} \Phi^{\beta} \Phi^{\gamma} \Phi^{\delta},
$$

where $d_{\alpha \beta \gamma \delta}$ is a rank-4 symmetric invariant tensor of $E_{7}$. Since the gauge symmetry is Abelian, the structure of the sigma model is essentially the same with the $Q^{N-2}$ case. Again, the constraint

$$
d_{\alpha \beta \gamma \delta} \varphi^{\alpha} \varphi^{\beta} F^{\gamma}=0
$$

defines a subspace in $E_{7} /\left[E_{6} \times U(1)\right]$ in the noncanonical branch.

\section{BOGOMOL'NYI-PRASAD-SOMMERFIELD STATES}

In this section, we study the BPS conditions in the nonlinear sigma models discussed in the previous section. BPS properties of Skyrme type and higher derivative models have been studied in various contexts [57-60]. It has been discussed that the gauged chiral models admit the $1 / 2$ BPS vortex state and the $1 / 4$ BPS states [29]. In the following, we derive the $1 / 2$ and $1 / 4$ BPS conditions in the nonlinear sigma models in the limit $g \rightarrow \infty$ of those in the gauged chiral models.

\section{A. BPS states in canonical branch}

In the canonical branch, the $1 / 2$ BPS vortex equation for the finite $g$ for gauged linear sigma model is

$$
\bar{D}_{z} \varphi^{i}=0, \quad \frac{1}{g} F_{12}-(\bar{\varphi} \varphi-\kappa \mathbf{1})=0,
$$

where $z=x^{1}+i x^{2}$ is the complex coordinate in the $\left(x^{1}, x^{2}\right)$-plane. These are $U(M)$ BPS semilocal vortex equations with $N$-flavors in the case of Grassmann case [55,61], for which we have found that higher derivative corrections do not appear. They reduce to the ordinary BPS vortex equations in the Abelian-Higgs model for the $U(1)$ gauge theory.

The energy bound for this configuration is $[29]^{3}$

$$
\mathcal{E}=-\kappa F_{12}^{0}
$$

\footnotetext{
${ }^{3}$ We comment on the positive semidefiniteness of the energy in the higher derivative chiral models. It is not always true that the energy density derived from Eq. (2.1) is positive semidefinite for arbitrary $\Lambda$ and the Kähler potential $K$. In order to define the BPS states as minima of energy, we have to choose appropriate $\Lambda$ and $K$ that makes the energy be positive semidefinite [27].
} 
The configuration is classified by the vortex number $\int d^{2} x F_{12}^{0}$.

In the nonlinear sigma model limit $g \rightarrow \infty$, the second condition in (5.1) gives the D-term constraint, while the first one becomes

$$
\bar{\partial}_{z} \varphi^{i}-\kappa^{-1}\left(\bar{\varphi}^{j} \bar{\partial}_{z} \varphi^{j}\right) \varphi^{i}=0 .
$$

This implies the following $1 / 2$ BPS lump equation in the sigma models:

$$
\bar{\partial}_{z} \varphi^{i}=0
$$

The bound (5.2) survives in the limit $g \rightarrow \infty$ and the result is

$$
\mathcal{E}=\frac{1}{2}\left|\partial_{z} \varphi\right|^{2}
$$

This provides the lump charge density in the nonlinear sigma model. We note that the derivative corrections never show up in Eq. (5.4) and the BPS bound (5.5) in the canonical branch. ${ }^{4}$

\section{B. BPS states in noncanonical branch}

In the noncanonical branch, we have only $1 / 4$ BPS state [29]. The BPS equations in the gauged linear sigma models are

$$
\bar{D}_{z} \varphi=-i F, \quad \frac{1}{g} F_{12}=\bar{\varphi} \varphi-\kappa \mathbf{1} .
$$

In the nonlinear sigma model limit $g \rightarrow \infty$, the BPS equations become

$$
\bar{D}_{z} \varphi=-i F, \quad \bar{\varphi} \varphi=\kappa \mathbf{1} .
$$

The latter equation gives the D-term constraint. From the first equation, we obtain

$$
\bar{D}_{z} \varphi D_{z} \bar{\varphi}=F \bar{F} .
$$

For the $\mathbb{C} P^{N-1}, Q^{N-2}$ cases, the gauge group is Abelian and we find an explicit solution for $F$. Then the BPS condition is rewritten as

\footnotetext{
${ }^{4}$ As denoted below Eq. (3.14), for the case of the $\mathbb{C} P^{N-1}$ model, the Lagrangian in Eq. (3.14) (with $\Lambda=$ const. can be obtained [49] as the low-energy effective theory of a BPS nonAbelian vortex in $\mathcal{N}=2$ supersymmetric $U(N)$ gauge theory [55]. In this context, $\mathbb{C} P^{N-1}$ lumps (sigma model instantons) on the vortex world sheet represent Yang-Mills instantons in the bulk theory, and such instanton-vortex composites are 1/4 BPS states [62]. It was discussed in Ref. [49] that this fact implies that lump solutions should not have derivative corrections.
}

$$
\overline{\tilde{D}}_{z} \bar{\varphi} \tilde{D}_{z} \varphi-\tilde{\tilde{D}}_{z} \varphi^{i} \tilde{D}_{z} \bar{\varphi}^{i}=\Lambda^{-1}(\varphi, \bar{\varphi}) .
$$

This is nothing but a compacton type equation. To see this, we choose an appropriate function $\Lambda$. For example, we consider the following function in the $\mathbb{C} P^{1}$ model:

$$
\Lambda=2\left(\frac{|u|^{2}}{1+|u|^{2}}\right)^{-\frac{s}{2}}
$$

where $s$ is a constant. Then, the BPS equation (5.9) results in the following compacton equation [45]:

$$
n g_{y}=-g^{\frac{s}{2}} .
$$

Here we have assumed the ansatz

$$
u=e^{i n \theta} f(r), \quad 1-g=\frac{1}{1+f^{2}}, \quad y=\frac{r^{2}}{2},
$$

where $r$ and $\theta$ are the polar coordinates in the $\left(x^{1}, x^{2}\right)$ plane.

\section{COMMENT ON FERMIONIC INTERACTIONS}

Finally, we comment on the fermionic interactions of the models, while in the previous sections, we have focused on the bosonic sector of the supersymmetric models and have shown that the vector field $A_{m}$ is integrated out exactly. However, the integration of the gaugino is rather awkward from the reason described below. We illustrate the problem in the $\mathbb{C} P^{N-1}$ model.

Assuming that $\Lambda$ does not contain $V$ for simplicity, the equation of motion for the vector superfield $V$ in the sigma model limit $g \rightarrow \infty$ is given by

$$
\begin{aligned}
& 2 \Phi^{i} e^{2 V} \Phi^{\dagger i}-\kappa+\frac{1}{4} \Lambda\left(\Phi, \Phi^{\dagger}\right) e^{4 V} \mathcal{D}^{\alpha} \Phi^{i} \mathcal{D}_{\alpha} \Phi^{j} \overline{\mathcal{D}}_{\dot{\alpha}} \Phi^{\dagger i} \overline{\mathcal{D}}^{\dot{\alpha}} \Phi^{\dagger j} \\
& +\frac{1}{4} D_{\alpha}\left[\Lambda\left(\Phi, \Phi^{\dagger}\right) e^{4 V} \Phi^{i} \mathcal{D}^{\alpha} \Phi^{j} \overline{\mathcal{D}}_{\dot{\alpha}} \Phi^{\dagger i} \overline{\mathcal{D}}^{\dot{\alpha}} \Phi^{\dagger j}\right] \\
& +\frac{1}{4} \bar{D}_{\dot{\alpha}}\left[\Lambda\left(\Phi, \Phi^{\dagger}\right) e^{4 V} \mathcal{D}_{\alpha} \Phi^{i} \mathcal{D}^{\alpha} \Phi^{j} \Phi^{\dagger i} \overline{\mathcal{D}}^{\dot{\alpha}} \Phi^{\dagger j}\right]=0 .
\end{aligned}
$$

If the term in the second line is ignored, we find that the vector superfield is solved by

$$
V=-\frac{1}{2} \log \left(2 \kappa^{-1} \Phi^{i} \Phi^{\dagger i}\right) .
$$

This is easily confirmed if one notices that the following relation holds for Eq. (6.2):

$$
\begin{aligned}
\Phi^{\dagger i} \mathcal{D}_{\alpha} \Phi^{i} & =\Phi^{\dagger i}\left[D_{\alpha} \Phi^{i}+2\left(D_{\alpha} V\right) \Phi^{i}\right] \\
& =\Phi^{\dagger i} D_{\alpha} \Phi^{i}-\frac{1}{|\Phi|^{2}} \Phi^{\dagger j} D_{\alpha} \Phi^{j}\left(\Phi^{i} \Phi^{\dagger i}\right)=0 .
\end{aligned}
$$


The would-be solution (6.2), whose $\theta \sigma^{m} \bar{\theta}$ component gives $A_{m}=i \kappa^{-1} \bar{\varphi}^{i} \partial_{m} \varphi^{i}$, precisely gives the correct Kähler potential $K=-\log \left(2 \kappa^{-1} \Phi \Phi^{\dagger}\right)$ for the $\mathbb{C} P^{N-1}$ model. However, when the term in the second line in Eq. (6.1) is included, Eq. (6.2) fails to be a solution. This implies that the expression in Eq. (6.2) is correct only in the bosonic sector. Including the second line in Eq. (6.1) modifies the equality in Eq. (6.2) in the fermionic sector.

We note that in solving the auxiliary field $F$, the fermionic fields $\psi$ in the chiral multiplets are introduced perturbatively around the bosonic solution $F=F(\varphi, \bar{\varphi})$ [20]. The same is true for the vector multiplet. Since the interaction terms are all expressed by the superfields explicitly, it is in principle possible to write down the equations for the fermions. We can integrate out the vector multiplet starting from the bosonic part of the solution (6.2) and introduce the fermions perturbatively. This procedure determines the fermionic interactions in the sigma model limit $g \rightarrow \infty$. Even though the fermionic interactions are rather cumbersome, we stress that the BPS conditions in the nonlinear sigma models are not affected by details of the fermionic interactions. As we have shown, this is obtained by those in the gauged chiral models.

\section{CONCLUSION AND DISCUSSIONS}

In this paper, we have constructed $\mathcal{N}=1$ supersymmetric higher derivative terms in nonlinear sigma models whose target spaces are Hermitian symmetric spaces. We have considered the sigma model limit of the supersymmetric gauged linear sigma models involving the derivative terms for chiral multiplets that are free from the auxiliary field problem and the Ostrogradski's ghost. In the sigma model limit, the vector multiplet does not propagate anymore and can be integrated out. Due to the supersymmetric derivative corrections, there are two distinct on-shell branches called the canonical and noncanonical branches. This structure is carried over to the nonlinear sigma models. We have shown that the gauge field $A_{m}$ is explicitly integrated out, both in the canonical and the noncanonical branches, even in the presence of higher derivative terms.

In the canonical branch, where the auxiliary field is given by $F=0$, we explicitly have written down the Lagrangians of the nonlinear sigma models. They consist of the canonical kinetic terms and the derivative corrections characterized by the arbitrary function $\Lambda$. This is a natural generalization of the sigma model construction discussed in Ref. [52].

For the $\mathbb{C} P^{N-1}$ model, we have obtained the supersymmetric $\mathbb{C} P^{N-1}$ Skyrme-Faddeev model. In the noncanonical branch, where the auxiliary field is given by $F \neq 0$, the situation changes drastically. For the $\mathbb{C} P^{N-1}$ model, an Abelian symmetry is gauged and we have found the explicit solutions of the nonzero auxiliary field $F$. We have solved all the constraints and have written down the explicit
Lagrangians of the $\mathbb{C} P^{N-1}$ nonlinear sigma model. The canonical quadratic kinetic term is absent in the noncanonical branch, and there is only the fourth derivative term as a generalization of the Skyrme-Faddeev term of the $\mathbb{C} P^{1}$ model. We thus have obtained the supersymmetric $\mathbb{C} P^{N-1}$ Skyrme-Faddeev model.

For the formulation of the $G_{M, N}$ model, a non-Abelian $U(N)$ symmetry is gauged in the gauged linear sigma model. Compared with the $\mathbb{C} P^{N-1}$ case for which the gauge symmetry is Abelian in the gauged linear sigma model, the equation of motion for the auxiliary field in the $G_{M, N}$ model is rather complicated. Although it is hard to find explicit $F \neq 0$ solutions, we have been able to integrate out the gauge field with the help of the gauge invariance of the higher derivative terms.

For other Hermitian symmetric spaces, in addition to D-term constraints, we further impose F-term constraints yielding holomorphic embedding of the target spaces into $\mathbb{C} P^{N-1}$ or Grassmann manifold, as was done for the case without higher derivative terms. In the canonical branches, these constraints are consistent in the presence of higher derivative terms, but we find that in the noncanonical branch these constraints yield further additional constraints reducing the target spaces to their submanifolds.

For the formulation of the $Q^{N-2}$ model, an Abelian symmetry is gauged in the gauged linear sigma model, but we have an extra constraint coming from the F-term embedding $Q^{N-2}$ to $\mathbb{C} P^{N-1}$. The nonzero solution $F \neq 0$ yields an extra constraint on the scalar fields. We have found that in order that these constraints are compatible with the equation of motion in the noncanonical branches, the dynamics of fields should be restricted to a subspace in the $Q^{N-2}$ manifold, which is a peculiar property of this model. We have also discussed the sigma models whose target spaces are $S O(2 N) / U(N), S p(N) / U(N)$, $E_{6} /[S O(10) \times U(1)]$, and $E_{7} /\left[E_{6} \times U(1)\right]$. The first two cases are essentially similar to the cases of the Grassmann and the $Q^{N-2}$ cases. For the latter two cases, although the superpotential is complicated compared with the $Q^{N-2}$ case, the structure of the higher derivative terms is similar to that of the $Q^{N-2}$ case. We also have found that these constructions provide the different sigma models even though there are several isomorphisms among the target spaces such as $G_{N, 1} \simeq G_{N, N-1} \simeq \mathbb{C} P^{N-1}, Q^{1} \simeq G_{2,1} \simeq \mathbb{C} P^{1}$, $Q^{4} \simeq G_{4,2}$, and so on.

Finally, we provide a comment on the BPS equations and the integration of the fermionic terms in the vector multiplet. In the canonical branches, BPS equations and their solutions are the same with those without higher derivative terms, while in the noncanonical branches they give compacton type configurations.

It would be interesting to study explicit solutions to the BPS equations in the noncanonical branch. As we have noted, the $1 / 4$ BPS equation in the noncanonical branch of 
the $\mathbb{C} P^{N-1}$ model reduces to that of the compactons. Finding solutions in the other sigma models is interesting. We will come back to these issues in future studies.

In this paper, we have considered the case that there are no potential terms in the nonlinear sigma models (although we have introduced superpotentials for the F-term constraints in the gauged linear sigma models). If we also introduce superpotentials or twisted masses (from dimensional reductions), there are more BPS states such as domain walls, their junctions, and so on [27]. Introducing potential terms and studying associated BPS states remain as a future work.

\section{ACKNOWLEDGMENTS}

This work was supported in part by Grant-in-Aid for Scientific Research, JSPS KAKENHI Grants No. JP18H01217 (M. N.) and No. JP20K03952 (S. S.).
[1] S. R. Coleman, J. Wess, and B. Zumino, Structure of phenomenological Lagrangians. 1., Phys. Rev. 177, 2239 (1969).

[2] C. G. Callan, Jr., S. R. Coleman, J. Wess, and B. Zumino, Structure of phenomenological Lagrangians. 2., Phys. Rev. 177, 2247 (1969).

[3] H. Leutwyler, On the foundations of chiral perturbation theory, Ann. Phys. (N.Y.) 235, 165 (1994).

[4] S. J. Gates, Jr., Why auxiliary fields matter: The strange case of the 4-D, $N=1$ supersymmetric QCD effective action, Phys. Lett. B 365, 132 (1996); Why auxiliary fields matter: The strange case of the 4-D, $N=1$ supersymmetric QCD effective action. 2., Nucl. Phys. B485, 145 (1997).

[5] S. J. Gates, Jr., M. T. Grisaru, M. E. Knutt, and S. Penati, The superspace WZNW action for 4-D, $N=1$ supersymmetric QCD, Phys. Lett. B 503, 349 (2001); S. J. Gates, Jr., M. T. Grisaru, M.E. Knutt, S. Penati, and H. Suzuki, Supersymmetric gauge anomaly with general homotopic paths, Nucl. Phys. B596, 315 (2001); S. J. Gates, Jr., M. T. Grisaru, and S. Penati, Holomorphy, minimal homotopy and the 4-D, $N=1$ supersymmetric Bardeen-Gross-Jackiw anomaly, Phys. Lett. B 481, 397 (2000).

[6] D. Nemeschansky and R. Rohm, Anomaly constraints on supersymmetric effective Lagrangians, Nucl. Phys. B249, 157 (1985); M. Nitta, A note on supersymmetric WZW term in four dimensions, Mod. Phys. Lett. A 15, 2327 (2000).

[7] E. A. Bergshoeff, R. I. Nepomechie, and H. J. Schnitzer, Supersymmetric skyrmions in four-dimensions, Nucl. Phys. B249, 93 (1985).

[8] L. Freyhult, The supersymmetric extension of the Faddeev model, Nucl. Phys. B681, 65 (2004).

[9] I. L. Buchbinder, S. Kuzenko, and Z. Yarevskaya, Supersymmetric effective potential: Superfield approach, Nucl. Phys. B411, 665 (1994).

[10] I. L. Buchbinder, S. M. Kuzenko, and A. Y. Petrov, Superfield chiral effective potential, Phys. Lett. B 321, 372 (1994).

[11] S. M. Kuzenko and S. J. Tyler, The one-loop effective potential of the Wess-Zumino model revisited, J. High Energy Phys. 09 (2014) 135.

[12] A. T. Banin, I. L. Buchbinder, and N. G. Pletnev, On quantum properties of the four-dimensional generic chiral superfield model, Phys. Rev. D 74, 045010 (2006).
[13] M. Gomes, J. R. Nascimento, A. Y. Petrov, and A. J. da Silva, On the effective potential in higher-derivative superfield theories, Phys. Lett. B 682, 229 (2009).

[14] F. S. Gama, M. Gomes, J. R. Nascimento, A. Y. Petrov, and A. J. da Silva, On the higher-derivative supersymmetric gauge theory, Phys. Rev. D 84, 045001 (2011).

[15] M. Rocek and A. A. Tseytlin, Partial breaking of global $D=4$ supersymmetry, constrained superfields, and threebrane actions, Phys. Rev. D 59, 106001 (1999).

[16] S. Sasaki, M. Yamaguchi, and D. Yokoyama, Supersymmetric DBI inflation, Phys. Lett. B 718, 1 (2012).

[17] C. Adam, J. M. Queiruga, J. Sanchez-Guillen, and A. Wereszczynski, Supersymmetric K field theories and defect structures, Phys. Rev. D 84, 065032 (2011).

[18] C. Adam, J. M. Queiruga, J. Sanchez-Guillen, and A. Wereszczynski, BPS bounds in supersymmetric extensions of K field theories, Phys. Rev. D 86, 105009 (2012).

[19] M. Ostrogradski, Memoires sur les equations differentielles relatives au probleme des isoperimetres, Mem. Ac. St. Petersbourg VI, 385 (1850).

[20] J. Khoury, J. L. Lehners, and B. Ovrut, Supersymmetric $P(X, \phi)$ and the ghost condensate, Phys. Rev. D 83, 125031 (2011).

[21] M. Koehn, J.-L. Lehners, and B. Ovrut, Ghost condensate in $N=1$ supergravity, Phys. Rev. D 87, 065022 (2013).

[22] J. Khoury, J.-L. Lehners, and B. A. Ovrut, Supersymmetric galileons, Phys. Rev. D 84, 043521 (2011).

[23] M. Koehn, J.-L. Lehners, and B. A. Ovrut, Higher-derivative chiral superfield actions coupled to $N=1$ supergravity, Phys. Rev. D 86, 085019 (2012).

[24] C. Adam, J. M. Queiruga, J. Sanchez-Guillen, and A. Wereszczynski, $N=1$ supersymmetric extension of the baby Skyrme model, Phys. Rev. D 84, 025008 (2011).

[25] C. Adam, J. M. Queiruga, J. Sanchez-Guillen, and A. Wereszczynski, Extended supersymmetry and BPS solutions in baby Skyrme models, J. High Energy Phys. 05 (2013) 108.

[26] F. Farakos and A. Kehagias, Emerging potentials in higherderivative gauged chiral models coupled to $N=1$ supergravity, J. High Energy Phys. 11 (2012) 077.

[27] M. Nitta and S. Sasaki, BPS states in supersymmetric chiral models with higher derivative terms, Phys. Rev. D 90, 105001 (2014). 
[28] M. Nitta and S. Sasaki, Higher derivative corrections to manifestly supersymmetric nonlinear realizations, Phys. Rev. D 90, 105002 (2014).

[29] M. Nitta and S. Sasaki, Classifying BPS states in supersymmetric gauge theories coupled to higher derivative chiral models, Phys. Rev. D 91, 125025 (2015).

[30] S. B. Gudnason, M. Nitta, and S. Sasaki, A supersymmetric Skyrme model, J. High Energy Phys. 02 (2016) 074; Topological solitons in the supersymmetric Skyrme model, J. High Energy Phys. 01 (2017) 014.

[31] J. M. Queiruga, Supersymmetric Galileons and auxiliary fields in $2+1$ dimensions, Phys. Rev. D 95, 125001 (2017).

[32] J. M. Queiruga and A. Wereszczynski, Non-uniqueness of the supersymmetric extension of the $O(3) \sigma$-model, J. High Energy Phys. 11 (2017) 141.

[33] T. Fujimori, M. Nitta, and Y. Yamada, Ghostbusters in higher derivative supersymmetric theories: Who is afraid of propagating auxiliary fields?, J. High Energy Phys. 09 (2016) 106; T. Fujimori, M. Nitta, K. Ohashi, and Y. Yamada, Ghostbusters in $f(R)$ supergravity, J. High Energy Phys. 05 (2018) 102.

[34] I. Antoniadis, E. Dudas, and D. M. Ghilencea, Supersymmetric models with higher dimensional operators, J. High Energy Phys. 03 (2008) 045.

[35] E. Dudas and D. M. Ghilencea, Effective operators in SUSY, superfield constraints and searches for a UV completion, J. High Energy Phys. 06 (2015) 124.

[36] M. Nitta, S. Sasaki, and R. Yokokura, Supersymmetry breaking in spatially modulated vacua, Phys. Rev. D 96, 105022 (2017); S. Bjarke Gudnason, M. Nitta, S. Sasaki, and R. Yokokura, Supersymmetry breaking and ghost Goldstino in modulated vacua, Phys. Rev. D 99, 045012 (2019).

[37] M. Nitta, S. Sasaki, and R. Yokokura, Spatially modulated vacua in a Lorentz-invariant scalar field theory, Eur. Phys. J. C 78, 754 (2018); S. B. Gudnason, M. Nitta, S. Sasaki, and R. Yokokura, Temporally, spatially, or lightlike modulated vacua in Lorentz invariant theories, Phys. Rev. D 99, 045011 (2019).

[38] T. Fujimori, M. Nitta, K. Ohashi, Y. Yamada, and R. Yokokura, Ghost-free vector superfield actions in supersymmetric higher-derivative theories, J. High Energy Phys. 09 (2017) 143.

[39] M. Nitta and R. Yokokura, Higher derivative three-form gauge theories and their supersymmetric extension, J. High Energy Phys. 10 (2018) 146; Topological couplings in higher derivative extensions of supersymmetric three-form gauge theories, J. High Energy Phys. 05 (2019) 102.

[40] S. Bolognesi and W. Zakrzewski, Baby Skyrme model, near-BPS approximations and supersymmetric extensions, Phys. Rev. D 91, 045034 (2015).

[41] J. M. Queiruga, Baby Skyrme model and fermionic zero modes, Phys. Rev. D 94, 065022 (2016).

[42] S. B. Gudnason, M. Barsanti, and S. Bolognesi, Near-BPS baby skyrmions, J. High Energy Phys. 11 (2020) 062.

[43] L. D. Faddeev and A. J. Niemi, Knots and particles, Nature (London) 387, 58 (1997).

[44] A. M. Polyakov and A. A. Belavin, Metastable states of twodimensional isotropic ferromagnets, Pis'ma Zh. Eksp. Teor. Fiz. 22, 503 (1975) [JETP Lett. 22, 245 (1975)].
[45] C. Adam, P. Klimas, J. Sanchez-Guillen, and A. Wereszczynski, Compact baby skyrmions, Phys. Rev. D 80, 105013 (2009); C. Adam, T. Romanczukiewicz, J. Sanchez-Guillen, and A. Wereszczynski, Investigation of restricted baby Skyrme models, Phys. Rev. D 81, 085007 (2010); C. Adam, J. Sanchez-Guillen, A. Wereszczynski, and W. J. Zakrzewski, Topological duality between vortices and planar skyrmions in BPS theories with area-preserving diffeomorphism symmetries, Phys. Rev. D 87, 027703 (2013); C. Adam, T. Romanczukiewicz, J. Sanchez-Guillen, and A. Wereszczynski, Magnetothermodynamics of BPS baby skyrmions, J. High Energy Phys. 11 (2014) 095.

[46] L. A. Ferreira and P. Klimas, Exact vortex solutions in a $C P^{N}$ Skyrme-Faddeev type model, J. High Energy Phys. 10 (2010) 008.

[47] L. A. Ferreira, P. Klimas, and W. J. Zakrzewski, Properties of some $(3+1)$ dimensional vortex solutions of the $C P^{N}$ model, Phys. Rev. D 84, 085022 (2011).

[48] L. A. Ferreira, P. Klimas, and W. J. Zakrzewski, Some properties of $(3+1)$ dimensional vortex solutions in the extended $C P^{N}$ Skyrme-Faddeev model, J. High Energy Phys. 12 (2011) 098.

[49] M. Eto, T. Fujimori, M. Nitta, K. Ohashi, and N. Sakai, Higher derivative corrections to non-Abelian vortex effective theory, Prog. Theor. Phys. 128, 67 (2012).

[50] J. Wess and J. Bagger, in Supersymmetry and Supergravity (Princeton University, Princeton, NJ, 1992), p. 259.

[51] S. M. Kuzenko, Non-compact duality, super-Weyl invariance and effective actions, J. High Energy Phys. 07 (2020) 222.

[52] K. Higashijima and M. Nitta, Supersymmetric nonlinear sigma models as gauge theories, Prog. Theor. Phys. 103, 635 (2000).

[53] A. D'Adda, P. Di Vecchia, and M. Luscher, Confinement and chiral symmetry breaking in $\mathrm{CP}^{\mathrm{n}-1}$ models with quarks, Nucl. Phys. B152, 125 (1979).

[54] S. Aoyama, The supersymmetric $U(N, r) \sigma$ model and its 0(2) extended supersymmetry, Nuovo Cimento A 57, 176 (1980).

[55] A. Hanany and D. Tong, Vortices, instantons and branes, J. High Energy Phys. 07 (2003) 037; R. Auzzi, S. Bolognesi, J. Evslin, K. Konishi, and A. Yung, Non-Abelian superconductors: Vortices and confinement in $N=2$ SQCD, Nucl. Phys. B673, 187 (2003); M. Eto, Y. Isozumi, M. Nitta, K. Ohashi, and N. Sakai, Moduli Space of Non-Abelian Vortices, Phys. Rev. Lett. 96, 161601 (2006); M. Eto, K. Konishi, G. Marmorini, M. Nitta, K. Ohashi, W. Vinci, and N. Yokoi, Non-Abelian vortices of higher winding numbers, Phys. Rev. D 74, 065021 (2006); M. Eto, Y. Isozumi, M. Nitta, K. Ohashi, and N. Sakai, Solitons in the Higgs phase: The moduli matrix approach, J. Phys. A 39, R315 (2006).

[56] K. Higashijima, T. Kimura, M. Nitta, and M. Tsuzuki, Large N limit of $N=2$ supersymmetric $Q^{N}$ model in twodimensions, Prog. Theor. Phys. 105, 261 (2001).

[57] C. Adam and A. Wereszczynski, BPS property and its breaking in $1+1$ dimensions, Phys. Rev. D 98, 116001 (2018).

[58] R. Casana, A. C. Santos, C. F. Farias, and A. L. Mota, Selfdual solitons in a generalized Chern-Simons baby Skyrme model, Phys. Rev. D 100, 045022 (2019). 
[59] R. Casana, A. C. Santos, C. F. Farias, and A. L. Mota, Selfdual solitons in a Maxwell-Chern-Simons baby Skyrme model, Phys. Rev. D 101, 045018 (2020).

[60] Ł. T. Stępień, Bogomolny equations for the BPS Skyrme models with impurity, J. High Energy Phys. 09 (2020) 140.

[61] M. Shifman and A. Yung, Non-Abelian semilocal strings in $N=2$ supersymmetric QCD, Phys. Rev. D 73, 125012 (2006); M. Eto, J. Evslin, K. Konishi, G. Marmorini, M. Nitta, K. Ohashi, W. Vinci, and N. Yokoi, On the moduli space of semilocal strings and lumps, Phys. Rev. D 76, 105002 (2007).
[62] A. Hanany and D. Tong, Vortex strings and fourdimensional gauge dynamics, J. High Energy Phys. 04 (2004) 066; M. Shifman and A. Yung, Non-Abelian string junctions as confined monopoles, Phys. Rev. D 70, 045004 (2004); M. Eto, Y. Isozumi, M. Nitta, K. Ohashi, and N. Sakai, Instantons in the Higgs phase, Phys. Rev. D 72, 025011 (2005); T. Fujimori, M. Nitta, K. Ohta, N. Sakai, and M. Yamazaki, Intersecting solitons, amoeba and tropical geometry, Phys. Rev. D 78, 105004 (2008). 